\title{
Trading performance for state constraint feasibility in stochastic constrained control: a randomized approach ${ }^{\text {th }}$
}

\author{
Luca Deori ${ }^{\mathrm{a}}$, Simone Garatti ${ }^{\mathrm{a}, *}$, Maria Prandini ${ }^{\mathrm{a}}$ \\ ${ }^{a}$ Dipartimento di Elettronica, Informazione e Bioingegneria, Politecnico di Milano, Piazza Leonardo da Vinci 32,20113 \\ Milano, Italy.
}

\begin{abstract}
Constrained control for stochastic linear systems is generally a difficult task due to the possible infeasibility of state constraints. In this paper, we focus on a finite control horizon and propose a design methodology where the constrained control problem is formulated as a chance-constrained optimization problem depending on some parameter. This parameter can be tuned so as to decide the appropriate trade-off between control cost minimization and state constraints satisfaction. An approximate solution is computed via a randomized algorithm. Precise guarantees about its feasibility for the original chance-constrained problem are provided. A numerical example shows the efficacy of the proposed methodology.
\end{abstract}

Keywords: Stochastic control, Constrained control, Randomized methods.

\section{Introduction}

In this paper, we address finite-horizon control for a stochastic linear system whose state $x_{t} \in \mathbb{R}^{n}$ evolves according to the equation

$$
x_{t+1}=A x_{t}+B u_{t}+B_{w} w_{t},
$$

where $u_{t} \in \mathbb{R}^{m}$ and $w_{t} \in \mathbb{R}^{n_{w}}$ represent the control input and a stochastic disturbance, respectively. Matrices $A, B, B_{w}$ have appropriate dimensions, and $B_{w}$ is assumed to be full rank. The disturbance $w_{t}$ has a possibly unbounded support and its actual value can be reconstructed from the state measurements through

$$
w_{t}=B_{w}^{\dagger}\left(x_{t+1}-A x_{t}-B u_{t}\right),
$$

where $B_{w}^{\dagger}$ denotes the pseudo-inverse of $B_{w}$.

Our goal is designing a state-feedback control policy so as to minimize some finite-horizon cost, while satisfying certain constraints on the control input and on the state. The proposed control policy can be applied over a receding horizon so as to obtain a Model Predictive Control (MPC) scheme, [1,2]. However, here we shall focus on the finite-horizon problem only, for which stability is actually not a concern.

A convenient parametrization of the control input $u_{t}$, which makes both the control and state variables affine in the design parameters $\gamma_{t} \in \mathbb{R}^{m}$ and $\theta_{t, j} \in \mathbb{R}^{m \times n_{w}}$, is given by

$$
u_{t}=\gamma_{t}+\sum_{j=0}^{t-1} \theta_{t, j} \varphi\left(w_{j}\right),
$$

\footnotetext{
"Research partly supported by the European Commission under the UnCoVerCPS project, grant number 643921.

${ }^{*}$ Corresponding author

Email address: simone.garatti@polimi.it (Simone Garatti)
} 
where $\varphi: \mathbb{R} \rightarrow \mathbb{R}$ is a given scalar function and the notation $\varphi\left(w_{j}\right)$ stands for $\varphi(\cdot)$ applied to each component of the $n_{w}$-dimensional vector $w_{j}{ }^{1}$. Policy (2) is indeed a state-feedback control policy since the disturbance is recovered from the state according to (1). Notably, if the scalar function $\varphi(\cdot)$ is the identity map (i.e., $\varphi(a)=a, \forall a \in \mathbb{R})$ and $B_{w}=I_{n}$, then, we obtain a policy that is equivalent to a feedback policy affine in the state [3. If $\varphi(\cdot)$ is given by the saturation function

$$
\varphi(a)= \begin{cases}-\bar{\varphi}, & a<-\bar{\varphi} \\ a, & |a| \leq \bar{\varphi} \\ \bar{\varphi}, & a>\bar{\varphi}\end{cases}
$$

(or alternatively by a sigmoidal function), then, the resulting policy is a nonlinear function of the state and provides a bounded input even if the disturbance is unbounded [4, 5].

The state $x_{t}$ and the control input $u_{t}$ are both random variables since they depend on the stochastic disturbance $w_{t}$. The system performance is then expressed in terms of an average cost function. More specifically, we adopt the average quadratic cost

$$
J=\mathbb{E}\left[\sum_{t=1}^{M} x_{t}^{T} Q_{t} x_{t}+\sum_{t=0}^{M-1} u_{t}^{T} R_{t} u_{t}\right]
$$

where $Q_{t} \in \mathbb{R}^{n \times n}$ and $R_{t} \in \mathbb{R}^{m \times m}$ are symmetric positive semidefinite matrices. We consider input and state constraints of the following form:

$$
\begin{array}{r}
\sup _{t \in\{0, \ldots, M-1\}}\left\|u_{t}\right\|_{\infty} \leq \bar{u}, \\
\sup _{t \in\{1, \ldots, M\}}\left\|C x_{t}\right\|_{\infty} \leq \bar{y} .
\end{array}
$$

Note that the value taken by the input and state variables along the reference finite-horizon $[0, M]$ is uncertain, since it depends on the noise process $w_{t}$ affecting the system evolution. To account for this when formulating the constraints, one can either enforce the constraints (5) to hold for every and each disturbance realization (hard constraints), even for those realizations that are quite unlikely to occur, or require them to hold only on a set of disturbance realizations of probability at least $1-\varepsilon$, with $\varepsilon \in(0,1)$ set by the user (soft constraints).

As for the control input, hard constraints are typically motivated by the presence of saturation limits of the actuators. However, they do not take into account the statistical properties of the noise and may lead to conservative solutions, which motivates the introduction of soft constraints on the input. Note that, whilst both hard and soft constraints on the input are always feasible (a feasible solution is obtained by setting all the design parameters equal to zero), hard constraints on the state are not feasible when the noise distribution has unbounded support, because $w_{t}$ enters additively the state equation and this contribution cannot be canceled through any control action. Hence, if the noise is not bounded, one can only head for soft state constraints, leading to the following two formulations for the input and state constraints:

- hard Es soft

$$
\left\{\begin{array}{l}
\sup _{t \in\{0, \ldots, M-1\}}\left\|u_{t}\right\|_{\infty} \leq \bar{u}, \forall\left(w_{0}, w_{1}, \ldots, w_{M-1}\right) \\
\mathbb{P}\left\{\sup _{t \in\{1, \ldots, M\}}\left\|C x_{t}\right\|_{\infty} \leq \bar{y}\right\} \geq 1-\varepsilon
\end{array}\right.
$$

\footnotetext{
${ }^{1}$ Note that one can also consider the more general setup where $n_{w}$ distinct $\varphi$ functions are used, one for each component of $w_{j}$. This allows for a better exploitation of the disturbance characteristics and, moreover, all the subsequent derivations can be straightforwardly extended to this setting as well. In the sequel, however, we will stick to the setup where the same $\varphi$ is applied to all the components of $w_{j}$ to avoid notational clutter.
} 
- soft $\mathcal{E} 3$ soft

$$
\mathbb{P}\left\{\sup _{t \in\{0, \ldots, M-1\}}\left\|u_{t}\right\|_{\infty} \leq \bar{u} \wedge \sup _{t \in\{1, \ldots, M\}}\left\|C x_{t}\right\|_{\infty} \leq \bar{y}\right\} \geq 1-\varepsilon .
$$

When formulating the constraints, the value of $\bar{y}$ in the state constraints is quite critical, because of the following two reasons:

- the feasibility of the soft constraint on the state is not always guaranteed since $\bar{y}$ may be not compatible with the disturbance characteristics, the system dynamics and initialization, and the saturation limits imposed to the control input;

- even when the soft constraint is feasible, the performance of the obtained solution can be too much adversely affected by the presence of the state constraints if $\bar{y}$ takes a conservative value.

As a remedy to prevent the critical issues above, rather than seeing $\bar{y}$ as a fixed value, one should try to modulate it so as to guarantee feasibility, while achieving the appropriate compromise between state constraint enforcement and performance.

In this paper we pursue this approach and, to address the feasibility issue, $\bar{y}$ is replaced by a decision variable, say $h$, so that it can be automatically set to a value compatible with the system dynamics and initialization, input constraints, and noise characteristics. Appropriate chance-constrained optimization problems depending on some parameter to be tuned are then introduced. In these optimization problems both state constraints and performance are accounted for, and the value for the parameter determines the trade-off between the objective of minimizing the control cost $J$ in (4) (performance) and that of minimizing $h$ (state constraint enforcement). By tuning this parameter, one can explore the different possible compromises between the two objectives - while preserving feasibility -, and choose the solution that is more satisfactory in terms of values achieved for $J$ and $h$.

From an algorithmic viewpoint, a randomized approach that is computationally tractable is proposed to provide an approximate solution to the resulting chance-constrained optimization problems. The proposed solution relies on the so-called scenario approach, 6, 7, 8, 9, 10, and requires using realizations of the disturbance $w_{t}$, which can be either available as time series data or artificially generated. In the latter case, the probability distribution of $w_{t}$ has to be known. A detailed analysis of the suitability of the method is also given.

A preliminary version of this work was presented as a conference contribution in 11]. The present paper significantly extends [11] in that detailed proofs of the results and a more comprehensive example are given.

Remark 1 (possible extensions). To avoid cumbersome notations, all the results in this paper are given for the constraints in (5). Nonetheless, the presented results are still valid in the more general setting of multiple affine constraints on $u$ and $x$,

$$
\begin{array}{ll}
\sup _{t \in\{0, \ldots, M-1\}}\left\|L_{k} u_{t}+g_{k}\right\|_{\infty} \leq \bar{u}_{k}, & k=1, \ldots, p_{u}, \\
\sup _{t \in\{1, \ldots, M\}}\left\|C_{k} x_{t}+d_{k}\right\|_{\infty} \leq \bar{y}_{k}, & k=1, \ldots, p_{x},
\end{array}
$$

through which one can e.g. pose distinct limits on the various input/state components. Also, as for the probabilistic constraints, extensions to the case when norms other than $\|\cdot\|_{\infty}$ are adopted can be easily worked out, the only requirement being that the norm argument is affine in the optimization variables. Furthermore, the proposed approach can also be adapted to the case when the disturbance is directly measurable and a feed-forward disturbance compensator is adopted.

\subsection{Brief literature overview}

We can distinguish two classes of approaches for stochastic constrained control depending on the relaxation method adopted to cope with probabilistic constraints, which can either be based on randomization 
or on some analytic approximation, possibly accounting for constraints indirectly vai a penalization term in the cost function.

Our approach belongs to the first class, as those proposed in [12, 13, 14. The closest approach to the present work is that in [14, where a penalty is added to the cost so as to avoid infeasibility, the key difference being that in 14 the disturbance is assumed to be bounded and hard constraints only are considered. Indeed, using penalty to ensure feasibility is a quite standard engineering practice in MPC 15. In all methods, limiting assumptions on the noise being bounded, or on the presence of input constraints only are made so that they are not applicable to our framework.

The approaches in 16, 17, 18, 19, 20, 21, 22, 23, 24, 25, belong to the second class and address problems where both input and state constraints are present and the disturbance has unbounded support, as it is the case in our paper. In particular, in [16, 19, 21, state constraints are accounted for by introducing a penalization term in the cost. In [17, 20, 22, 24, 25, an analytic convex relaxation of chance constraints is adopted, while in [18, 23. the support of the disturbance is reduced to a compact set by suitably cutting the tails of the disturbance distribution and then providing a result that holds with a certain (high) probability. In all these analytic approaches that do not resort to randomization, the disturbance is assumed to be a sequence of independent and identically distributed (i.i.d.) random variables. The approaches [16, 17, 18, 20, 22, 24 also require the disturbance to be Gaussian.

In most of the cited approaches, a receding horizon implementation of the finite horizon solution to the stochastic constrained optimization is adopted, leading to stochastic MPC solutions. This brings into the picture further relevant issues like recursive feasibility and stability (see e.g. the recent surveys [26] and 27. On stochastic MPC) that, however, go beyond the scope of this paper. Also, stability may be even impossible to achieve in our setting where the control input is bounded and the disturbance is unbounded as discussed in 28] with reference to mean square stability.

Interestingly, constraint tightening can be instrumental to enforce recursive feasibility and stability in stochastic MPC, as shown in [29] though with reference to the case of an i.i.d. disturbance sequence with bounded and convex support. The analysis is performed assuming that suitably designed probabilistic constraints are satisfied. Either randomized or analytic methods can then be used for the algorithmic solution of the involved chance-constrained optimization problem.

\subsection{Structure of the paper}

Section 2 introduces compact notations to simplify the reading. Two approaches to trade $J$ with respect to $h$ are presented in Section 3, while Section 4 introduces suitable relaxations to the resulting chanceconstrained optimization problems leading to computationally tractable problems. Section 5 focuses on the tuning of the parameter affecting the compromise between performance and state constraint enforcement in both approaches. A thorough discussion on the properties of the achieved approximate solutions and on the relation between the two approaches is also provided. The related proofs are presented in Section 6. A numerical example is presented in Section 7, while some concluding remarks are drawn in Section 8 .

\section{Notations}

In this section we introduce compact notations to simplify the equations and ease the paper understanding.

Let

$$
\mathbf{x}=\left[\begin{array}{c}
x_{1} \\
x_{2} \\
\vdots \\
x_{M}
\end{array}\right] \quad \mathbf{u}=\left[\begin{array}{c}
u_{0} \\
u_{1} \\
\vdots \\
u_{M-1}
\end{array}\right] \quad \mathbf{w}=\left[\begin{array}{c}
w_{0} \\
w_{1} \\
\vdots \\
w_{M-1}
\end{array}\right]
$$

be the vectors collecting state, input, and disturbance samples along the reference time-horizon. Then, it is easy to show that

$$
\begin{aligned}
& \mathbf{x}=\mathbf{F} x_{0}+\mathbf{G u}+\mathbf{H w} \\
& \mathbf{u}=\Gamma+\Theta \varphi(\mathbf{w})
\end{aligned}
$$


where matrices $\mathbf{F}, \mathbf{G}$ and $\mathbf{H}$ are given by

$$
\begin{gathered}
\mathbf{F}=\left[\begin{array}{c}
A \\
A^{2} \\
\vdots \\
A^{M}
\end{array}\right] \quad \mathbf{G}=\left[\begin{array}{cccc}
B & 0_{n \times m} & \cdots & 0_{n \times m} \\
A B & B & \ddots & \vdots \\
\vdots & \ddots & \ddots & 0_{n \times m} \\
A^{M-1} B & \cdots & A B & B
\end{array}\right] \\
\mathbf{H}=\left[\begin{array}{cccc}
B_{w} & 0_{n \times n_{w}} & \cdots & 0_{n \times n_{w}} \\
A B_{w} & B_{w} & \ddots & \vdots \\
\vdots & \ddots & \ddots & 0_{n \times n_{w}} \\
A^{M-1} B_{w} & \cdots & A B_{w} & B_{w}
\end{array}\right]
\end{gathered}
$$

whereas $\Gamma$ and $\Theta$ are given by

$$
\Gamma=\left[\begin{array}{c}
\gamma_{0} \\
\gamma_{1} \\
\vdots \\
\gamma_{M-1}
\end{array}\right] \Theta=\left[\begin{array}{cccc}
0_{m \times n_{w}} & 0_{m \times n_{w}} & \cdots & 0_{m \times n_{w}} \\
\theta_{1,0} & 0_{m \times n_{w}} & \ddots & \vdots \\
\vdots & \ddots & \ddots & 0_{m \times n_{w}} \\
\theta_{M-1,0} & \cdots & \theta_{M-1, M-2} & 0_{m \times n_{w}}
\end{array}\right]
$$

From (6) it is clear that both the state and control input depend linearly on the parameters $\Gamma$ and $\Theta$. For ease of notation, we do not make this dependence explicit and use $\mathbf{u}$ and $\mathbf{x}$ in place of $\mathbf{u}(\Gamma, \Theta)$ and $\mathbf{x}(\Gamma, \Theta)$.

If we set

$$
\mathbf{Q}=\left[\begin{array}{ccc}
Q_{1} & \cdots & 0_{n \times n} \\
\vdots & \ddots & \vdots \\
0_{n \times n} & \cdots & Q_{M}
\end{array}\right] \quad \mathbf{R}=\left[\begin{array}{ccc}
R_{0} & \cdots & 0_{m \times m} \\
\vdots & \ddots & \vdots \\
0_{m \times m} & \cdots & R_{M-1}
\end{array}\right]
$$

$m_{\mathbf{w}}=\mathbb{E}[\mathbf{w}], m_{\varphi}=\mathbb{E}[\varphi(\mathbf{w})]$ and

$$
\mathbf{V}=\left[\begin{array}{cc}
V_{\varphi \varphi} & V_{\varphi \mathbf{w}} \\
V_{\varphi \mathbf{w}}^{T} & V_{\mathbf{w w}}
\end{array}\right]
$$

where $V_{\mathbf{w w}}$ and $V_{\varphi \varphi}$ are the covariance matrices of $\mathbf{w}$ and $\varphi(\mathbf{w})$ and $V_{\varphi \mathbf{w}}$ is the cross covariance matrix of $\varphi(\mathbf{w})$ and $\mathbf{w}$; then, the control cost (4) can be expressed as the following convex function of $(\Gamma, \Theta)$ :

$$
\begin{aligned}
& J(\Gamma, \Theta)=\quad \mathbb{E}\left[\mathbf{x}^{T} \mathbf{Q} \mathbf{x}+\mathbf{u}^{T} \mathbf{R u}\right] \\
& =\left(\mathbf{F} x_{0}+\mathbf{G} \Gamma+\mathbf{G} \Theta m_{\varphi}+\mathbf{H} m_{\mathbf{w}}\right)^{T} \mathbf{Q}\left(\mathbf{F} x_{0}+\mathbf{G} \Gamma+\mathbf{G} \Theta m_{\varphi}+\mathbf{H} m_{\mathbf{w}}\right) \\
& +\operatorname{tr}\left(\mathbf{Q}^{\frac{1}{2}} \mathbf{G} \Theta V_{\varphi \varphi} \Theta^{T} \mathbf{G}^{T} \mathbf{Q}^{\frac{1}{2}}\right)+\operatorname{tr}\left(\mathbf{Q}^{\frac{1}{2}} \mathbf{H} V_{\mathbf{w} \mathbf{w}} \mathbf{H}^{T} \mathbf{Q}^{\frac{1}{2}}\right)+2 \operatorname{tr}\left(\mathbf{Q}^{\frac{1}{2}} \mathbf{G} \Theta V_{\varphi \mathbf{w}} \mathbf{H}^{T} \mathbf{Q}^{\frac{1}{2}}\right) \\
& +\left(\Gamma+\Theta m_{\varphi}\right)^{T} \mathbf{R}\left(\Gamma+\Theta m_{\varphi}\right)+\operatorname{tr}\left(\mathbf{R}^{\frac{1}{2}} \Theta V_{\varphi \varphi} \Theta^{T} \mathbf{R}^{\frac{1}{2}}\right) \\
& =\left(\mathbf{F} x_{0}+\mathbf{G} \Gamma+\mathbf{G} \Theta m_{\varphi}+\mathbf{H} m_{\mathbf{w}}\right)^{T} \mathbf{Q}\left(\mathbf{F} x_{0}+\mathbf{G} \Gamma+\mathbf{G} \Theta m_{\varphi}+\mathbf{H} m_{\mathbf{w}}\right)
\end{aligned}
$$

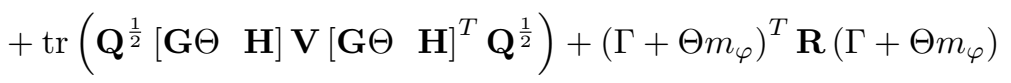

$$
\begin{aligned}
& +\operatorname{tr}\left(\mathbf{R}^{\frac{1}{2}} \Theta V_{\varphi \varphi} \Theta^{T} \mathbf{R}^{\frac{1}{2}}\right)
\end{aligned}
$$

As for the constraints (5), if we set

$$
\mathbf{C}=\left[\begin{array}{cccc}
C & 0 & \ldots & 0 \\
0 & C & & \vdots \\
\vdots & & \ddots & 0 \\
0 & \ldots & 0 & C
\end{array}\right]
$$


then, they can be expressed in compact form as follows:

$$
\begin{aligned}
& \|\mathbf{u}\|_{\infty} \leq \bar{u} \\
& \|\mathbf{C x}\|_{\infty} \leq h,
\end{aligned}
$$

where $\bar{y}$ has been replaced with the optimization variable $h$.

Thanks to the linear dependence of $\mathbf{u}$ and $\mathbf{x}$ on $\Gamma$ and $\Theta$, these constraints turn out to be convex with respect to the optimization variables, $h, \Gamma, \Theta$.

\section{Trading performance for state constraint feasibility}

In this section, two parametric optimization problems that account for the modulation of performance in favor of the enforcement of state constraints are introduced. As anticipated in the introduction, in both problems the bound $h$ on the norm of the state is regarded as an optimization variable so that feasibility is always recovered.

\subsection{Additional penalization term in the control cost}

In this first method, a penalization term is added to the average quadratic cost $J$ in (4) in order to penalize too high values for $h$ :

$$
J^{\prime}=J+\mu h .
$$

The coefficient $\mu \geq 0$ is the relative weight between $J$ and $h$ and determines the trade-off between the objective of having a small $J$ and that of having a small $h$. Depending on the kind of constraint adopted for the control input, two chance-constrained problems can be formulated:

$$
\begin{gathered}
\min _{\Gamma, \Theta, h} J(\Gamma, \Theta)+\mu h \quad \text { subject to: } \\
\left\{\begin{array}{l}
\|\mathbf{u}\|_{\infty} \leq \bar{u}, \forall \mathbf{w} \\
\mathbb{P}\left\{\|\mathbf{C x}\|_{\infty} \leq h\right\} \geq 1-\varepsilon
\end{array}\right. \\
\min _{\Gamma, \Theta, h} J(\Gamma, \Theta)+\mu h \quad \text { subject to: } \\
\mathbb{P}\left\{\|\mathbf{u}\|_{\infty} \leq \bar{u} \wedge\|\mathbf{C x}\|_{\infty} \leq h\right\} \geq 1-\varepsilon
\end{gathered}
$$

\subsection{Two-step approach based on a pre-defined admissible deterioration of the control cost}

In this second approach, the two objectives of minimizing the control cost as well as the bound $h$ on the state are handled by solving two optimization problems in cascade. In the first one, the smallest admissible control cost is found by minimizing it subject to the control input constraints only, whereas in the second one, $h$ is minimized subject to the constraints on both state and control input and a further constraint on the maximum admissible degradation of the control cost with respect to the value $J^{\star}$ computed in the first problem: $J \leq J^{\star}+\alpha$, with $\alpha \geq 0$. Again, the coefficient $\alpha$ determines the trade-off between performance and state constraint enforcement.

When hard constraints are imposed on the control input, the first optimization problem is given by

$$
\begin{aligned}
& \min _{\Gamma, \Theta} J(\Gamma, \Theta) \quad \text { subject to: } \\
& \|\mathbf{u}\|_{\infty} \leq \bar{u} \quad \forall \mathbf{w},
\end{aligned}
$$

while, letting $J^{\star}$ be the optimal cost obtained by solving 11a), the second optimization problem is:

$$
\begin{aligned}
& \min _{\Gamma, \Theta, h} h \quad \text { subject to: } \\
& \left\{\begin{array}{l}
\|\mathbf{u}\|_{\infty} \leq \bar{u} \quad \forall \mathbf{w} \\
\mathbb{P}\left\{\|\mathbf{C x}\|_{\infty} \leq h\right\} \geq 1-\varepsilon . \\
J(\Gamma, \Theta) \leq J^{\star}+\alpha
\end{array}\right.
\end{aligned}
$$


If the control input is subject to a probabilistic constraint as well, then, the first optimization problem writes

$$
\begin{gathered}
\min _{\Gamma, \Theta} J(\Gamma, \Theta) \text { subject to: } \\
\mathbb{P}\left\{\|\mathbf{u}\|_{\infty} \leq \bar{u}\right\} \geq 1-\varepsilon,
\end{gathered}
$$

while, letting $J^{\star}$ denote the optimal cost obtained by solving 12 a , the second optimization problem is:

$$
\begin{aligned}
& \min _{\Gamma, \Theta, h} h \quad \text { subject to: } \\
& \left\{\begin{array}{l}
\mathbb{P}\left\{\|\mathbf{u}\|_{\infty} \leq \bar{u} \wedge\|\mathbf{C} \mathbf{x}\|_{\infty} \leq h\right\} \geq 1-\varepsilon \\
J(\Gamma, \Theta) \leq J^{\star}+\alpha
\end{array}\right.
\end{aligned}
$$

Remark 2. As is clear, also a two-step approach where the role of $J$ and $h$ is inverted (namely in the first step the smallest possible value for $h$ subject to input and state constraints is found while in the second step the control cost is minimized subject to an additional constraint on the maximum admissible degradation for h) can be considered. However, it gives no real advantage over the two presented approaches and, as it will be explained later in Remark 4, this approach suffers from some drawbacks in its approximate resolution. Henceforth, it will not be taken into account.

Remark 3. In both the two approaches, $\mu$ and $\alpha$ are tuning parameters through which one can tradeoff between contrasting objectives. In the two-step approach, $\alpha$ has a precise meaning, namely, it is the maximum allowed degradation of the cost $J$ with respect to $J^{\star}$ and this interpretation helps the user to select the most proper value of $\alpha$ for the problem at hand. There is not, instead, a similar interpretation for $\mu$, being its effect on $J$ and $h$ much more indirect. As such, a proper tuning of $\mu$ may be more difficult to achieve, and usually it requires trials and errors. On the other hand, the approach with the additional penalization term in the cost has some advantages as far as its approximate resolution is concerned, as it will be shown in the next section.

\section{Approximate solution to the optimization problems}

The resolution of problems $(9-12$ posed in Sections 3.1 and 3.2 demands for algorithmic methods to tackle the robust and probabilistic constraints.

As for the robust constraint $\|\mathbf{u}\|_{\infty} \leq \bar{u}, \forall \mathbf{w}$, being the support of $\mathbf{w}$ unbounded, function $\varphi(\cdot)$ has to be chosen as a saturation function as in (3) because otherwise, if e.g. $\varphi(\cdot)$ is the identity map, the robust constraint would always lead to solutions with $\Theta=0$, i.e., to a control policy without the feedback term. Assuming that $|\varphi(\cdot)| \leq \bar{\varphi}$ and following [4, 5, the robust constraint can be then replaced by the following finite set of convex constraints

$$
\left|\Gamma_{i}\right|+\left\|\Theta_{i}\right\|_{1} \bar{\varphi} \leq \bar{u}, i=1, \ldots, m M
$$

where $\Gamma_{i}$ denotes the $i$-th element of vector $\Gamma$ and $\Theta_{i}$ the $i$-th row of $\Theta$. The idea behind $(13)$ is that $\mathbf{u}$ cannot be worse than when the components of $\varphi(\mathbf{w})$ have all absolute value equal to $\bar{\varphi}$ and signs such that the elements of each row $\Theta_{i} \varphi(\mathbf{w})+\Gamma_{i}$ positively sum up. Plainly, any feasible point for 13 is also feasible for the original robust constraint, and, moreover, they are equivalent as long as $|\varphi(\mathbf{w})|=\bar{\varphi}$ for some $\mathbf{w}$. The number of constraints in $(13)$ is finite and usually small, and 13 can be dealt with by means of standard solvers.

The probabilistic constraints

$$
\mathbb{P}\left\{\|\mathbf{C x}\|_{\infty} \leq h\right\} \geq 1-\varepsilon \quad \text { and } \quad \mathbb{P}\left\{\|\mathbf{u}\|_{\infty} \leq \bar{u} \wedge\|\mathbf{C x}\|_{\infty} \leq h\right\} \geq 1-\varepsilon,
$$


instead, are much harder to solve because they may be even non-convex, though $\|\mathbf{u}\|_{\infty} \leq \bar{u}$ and $\|\mathbf{C x}\|_{\infty} \leq h$ are convex for any fixed realization of $\mathbf{w}$. An exact resolution of the problems $(9)-(12)$ is therefore not possible, except for few special cases, and some level of approximation must be accepted.

In the remainder of this section, suitable relaxations of the probabilistic constraints $\mathbb{P}\left\{\|\mathbf{C x}(\mathbf{w})\|_{\infty} \leq h\right\} \geq$ $1-\varepsilon$ and $\mathbb{P}\left\{\|\mathbf{u}\|_{\infty} \leq \bar{u} \wedge\|\mathbf{C x}\|_{\infty} \leq h\right\} \geq 1-\varepsilon$ are introduced and discussed so as to reformulate problems (9)-12 in a way that is amenable of resolution by means of standard convex optimization techniques.

\subsection{Algorithms}

Probabilistic constraints are tacked by resorting to the scenario approach, a recently developed randomized method to approximately solve chance-constrained problems [6, 7, 8, 9, 10].

The idea behind the scenario approach is very simple. A set of $N$ realizations, i.e. scenarios, of the disturbance $\mathbf{w}$, say $\mathbf{w}^{(1)}, \mathbf{w}^{(2)}, \ldots, \mathbf{w}^{(N)}$, is generated according to the underlying probability distribution of $\mathbf{w}$. Then, the probabilistic constraints are replaced with a finite number $N$ of constraints of the type $\|\mathbf{C x}\|_{\infty} \leq h$ and/or $\|\mathbf{u}\|_{\infty} \leq \bar{u}$, those obtained in correspondence of the generated instances of the disturbance. More precisely, writing explicitly the dependence of $\mathbf{x}$ and $\mathbf{u}$ on $\mathbf{w}$, the constraint

$$
\mathbb{P}\left\{\|\mathbf{C x}(\mathbf{w})\|_{\infty} \leq h\right\} \geq 1-\varepsilon
$$

is replaced by

$$
\left\|\mathbf{C x}\left(\mathbf{w}^{(i)}\right)\right\|_{\infty} \leq h, \quad i=1, \ldots, N,
$$

while

is replaced by

$$
\mathbb{P}\left\{\|\mathbf{u}\|_{\infty} \leq \bar{u} \wedge\|\mathbf{C x}\|_{\infty} \leq h\right\} \geq 1-\varepsilon
$$

$$
\left\{\begin{array}{l}
\left\|\mathbf{u}\left(\mathbf{w}^{(i)}\right)\right\|_{\infty} \leq \bar{u} \\
\left\|\mathbf{C x}\left(\mathbf{w}^{(i)}\right)\right\|_{\infty} \leq h
\end{array} \quad i=1, \ldots, N .\right.
$$

Summarizing, depending on the chosen type of constraint (robust or in probability) for the input, and on the chosen method to take into account the presence of the optimization variable $h$, the possible reformulations of $9-12$ are the following:

- Additional penalization term and hard constraint on input:

$$
\begin{aligned}
& \min _{\Gamma, \Theta, h} J(\Gamma, \Theta)+\mu h \quad \text { subject to: } \\
& \left\{\begin{array}{l}
\left|\Gamma_{i}\right|+\left\|\Theta_{i}\right\|_{1} \bar{\varphi} \leq \bar{u}, \quad i=1, \ldots, m M \\
\left\|\mathbf{C x}\left(\mathbf{w}^{(i)}\right)\right\|_{\infty} \leq h, \quad i=1, \ldots, N
\end{array}\right.
\end{aligned}
$$

- Additional penalization term and soft constraint on input:

$$
\begin{aligned}
& \min _{\Gamma, \Theta, h} J(\Gamma, \Theta)+\mu h \quad \text { subject to: } \\
& \left\{\begin{array}{l}
\left\|\mathbf{u}\left(\mathbf{w}^{(i)}\right)\right\|_{\infty} \leq \bar{u} \\
\left\|\mathbf{C x}\left(\mathbf{w}^{(i)}\right)\right\|_{\infty} \leq h
\end{array} \quad i=1, \ldots, N\right.
\end{aligned}
$$

- Two-step approach and hard constraint on input:

$$
\begin{aligned}
& \min _{\Gamma, \Theta} J(\Gamma, \Theta) \quad \text { subject to: } \\
& \left|\Gamma_{i}\right|+\left\|\Theta_{i}\right\|_{1} \bar{\varphi} \leq \bar{u}, i=1, \ldots, m M
\end{aligned}
$$


Let $J^{\star}$ be the optimal cost value of $16 \mathrm{a}$ ).

$$
\begin{aligned}
& \min _{\Gamma, \Theta, h} h \quad \text { subject to: } \\
& \left\{\begin{array}{l}
\left|\Gamma_{i}\right|+\left\|\Theta_{i}\right\|_{1} \bar{\varphi} \leq \bar{u}, \quad i=1, \ldots, m M, \\
\left\|\mathbf{C x}\left(\mathbf{w}^{(i)}\right)\right\|_{\infty} \leq h, \quad i=1, \ldots, N \\
J(\Gamma, \Theta) \leq J^{\star}+\alpha
\end{array}\right.
\end{aligned}
$$

- Two-step approach and soft constraint on input:

$$
\begin{aligned}
& \min _{\Gamma, \Theta} J(\Gamma, \Theta) \quad \text { subject to: } \\
& \left\|\mathbf{u}\left(\mathbf{w}^{(i)}\right)\right\|_{\infty} \leq \bar{u} \quad i=1, \ldots, N
\end{aligned}
$$

Let $J^{\star}$ be the optimal cost value of $17 \mathrm{a}$.

$$
\begin{aligned}
& \min _{\Gamma, \Theta, h} h \quad \text { subject to: } \\
& \left\{\begin{array}{l}
\left\{\begin{array}{l}
\left\|\mathbf{u}\left(\mathbf{w}^{(i)}\right)\right\|_{\infty} \leq \bar{u} \\
\left\|\mathbf{C x}\left(\mathbf{w}^{(i)}\right)\right\|_{\infty} \leq h \\
J(\Gamma, \Theta) \leq J^{\star}+\alpha
\end{array} \quad i=1, \ldots, N\right.
\end{array}\right.
\end{aligned}
$$

Note that all the relaxed optimization problems above are always feasible (just take $\Gamma=0, \Theta=0$ and $\left.h=\max _{i}\left\|\mathbf{C x}\left(\mathbf{w}^{(i)}\right)\right\|_{\infty}\right)$. To this purpose, note that in the two-step approach with soft constraints on input the same realizations $\mathbf{w}^{(1)}, \mathbf{w}^{(2)}, \ldots \mathbf{w}^{(N)}$ must be used both in $(17 \mathrm{a})$ and in $(17 \mathrm{~b})$, because, otherwise, the feasibility of the optimization problem (17b), whose constraints depend on the solution of (17a), may be compromised.

The resolution of (14)-(17) amounts to solving convex programs with a finite number of constraints and requires no machinery other than standard convex optimization solvers like those used by CVX [30, and YALMIP [31.

Despite the apparent naivety of the scenario approach, the obtained solutions come with precise guarantees about their feasibility with respect the original probabilistic constraints as long as $N$ is suitably chosen. This is discussed in the next section.

\subsection{Chance-constrained feasibility of the obtained approximate solutions}

The problems (14)- (17) are obtained as relaxations of the original problems $(9)-(12)$. The sub-optimality of the obtained solutions is the price we must pay to enhance computational tractability. However, a main issue is whether the solutions to problems $(14), \sqrt{15}, \sqrt{16})$, and $(17)$ are feasible for the original constraints on $\mathbf{u}$ and $\mathbf{x}$ in problems $(9),(10),(11)$, and (12), respectively.

As already discussed, the reformulation introduced for the robust constraint, see $(13)$, is such that feasibility with respect to the original hard constraint is preserved. It is a fact that a similar result holds for the relaxation of the constraints in probability introduced by the scenario approach, though this is much less evident than the previous case. The following theorem taken from 8 provides the fundamental result in this respect.

Theorem 1. Let $f(\xi): \mathbb{R}^{d} \rightarrow \mathbb{R}$ be a convex function and $g(\xi, \delta): \mathbb{R}^{d} \times \Delta \rightarrow \mathbb{R}$ be a parametric family of convex functions (i.e. $g(\xi, \delta)$ is convex in $\xi$ for any fixed value of $\delta \in \Delta$ ). Moreover, let $\Xi$ be any given convex subset of $\mathbb{R}^{d}$. For a given positive integer $N$, consider the optimization problem

$$
\begin{aligned}
& \min _{\xi \in \Xi \subseteq \mathbb{R}^{d}} f(\xi) \quad \text { subject to: } \\
& g\left(\xi, \delta^{(i)}\right) \leq 0, \quad i=1, \ldots, N,
\end{aligned}
$$


where $\delta^{(1)}, \delta^{(2)}, \ldots, \delta^{(N)}$ are samples independently extracted according to a given probability $\mathbb{P}$ over $\Delta$, and let $\xi^{*}$ be the solution. If multiple solutions arise, then $\xi^{*}$ denotes the one which minimizes $\left\|\xi^{*}-\bar{\xi}\right\|$, where $\bar{\xi}$ is a user-chosen reference point.

For any $\varepsilon \in(0,1)$ and $\beta \in(0,1)$, if

$$
N \geq \frac{d+1+\ln (1 / \beta)+\sqrt{2(d+1) \ln (1 / \beta)}}{\varepsilon},
$$

then $\xi^{*}$ is feasible for the constraint in probability

$$
\mathbb{P}\{g(\xi, \delta) \leq 0\} \geq 1-\varepsilon,
$$

with confidence greater than or equal to $1-\beta$.

Theorem 1 was proven in $[8$, although in [8] an implicit expression for $N$ is provided. The explicit expression (18) was derived in [32] and [33]. Theorem 1 is amenable of extensions, like e.g. when some scenario constraints are removed [34, or when the scenario constraints are not convex [35, 36]. This extensions, however, are not considered here.

Note that the feasibility of $\xi^{*}$ for the probabilistic constraint can be guaranteed with high confidence $1-\beta$ only. This is intrinsically so because $\xi^{*}$ is random as it depends on the extracted $\delta^{(1)}, \delta^{(2)}, \ldots, \delta^{(N)}$. However, $N$ depends on $\beta$ logarithmically so that small values of $\beta$, like $\beta=10^{-5}$ or $\beta=10^{-7}$, can be forced in without affecting $N$ too much. With such small values for $\beta$, the result in Theorem 1 on the feasibility of $\xi^{*}$ for the constraint in probability holds in practice with probability 1 .

By letting $\xi=(\Gamma, \Theta, h), \delta=\mathbf{w}$,

$$
\begin{aligned}
& f(\xi)= \begin{cases}J(\Gamma, \Theta)+\mu h & \text { for } 14,, 15) \\
h & \text { for } 16 \mathrm{~b})\end{cases} \\
& g\left(\xi, \delta^{(i)}\right)=\left\{\begin{array}{ll}
\left\|\mathbf{C x}\left(\mathbf{w}^{(i)}\right)\right\|_{\infty}-h & \text { for } 14 \\
\max \left\{\left\|\mathbf{u}\left(\mathbf{w}^{(i)}\right)\right\|_{\infty}-\bar{u},\left\|\mathbf{C x}\left(\mathbf{w}^{(i)}\right)\right\|_{\infty}-h\right\} & \text { for } 15
\end{array},\right.
\end{aligned}
$$

and $\Xi$ be the intersection of the other constraints that do not depend on $\mathbf{w}^{(i)}$, a direct application of Theorem 1 to (14), (15), (16b) shows that, if $N$ satisfies (18), then the solutions of Problems (14), (15), (16) are feasible with high confidence $1-\beta$ for the constraints on $\mathbf{u}$ and $\mathbf{x}$ in (9), (10), (11), respectively.

As for Problem (17), Theorem 1 does not apply in this case. As a matter of fact, $J^{\star}$ in (17b) should be more properly written as $J^{\star}\left(\mathbf{w}^{(1)}, \mathbf{w}^{(2)}, \ldots, \mathbf{w}^{(N)}\right)$, being obtained as the optimal value of (17a), a program where constraints depend on $\mathbf{w}^{(1)}, \mathbf{w}^{(2)}, \ldots, \mathbf{w}^{(N)}$. This means that $\Xi=\Xi\left(\mathbf{w}^{(1)}, \mathbf{w}^{(2)}, \ldots, \mathbf{w}^{(N)}\right)$, a setup which is not covered by Theorem 1. Although we experimentally verified that, for $N$ large enough, the solution of Problem (17) is usually feasible for the constraint in probability

$$
\mathbb{P}\left\{\|\mathbf{u}\|_{\infty} \leq \bar{u} \wedge\|\mathbf{C x}\|_{\infty} \leq h\right\} \geq 1-\varepsilon
$$

(see e.g. the numerical example in Section 7), we were not able to prove that feasibility holds with high confidence $1-\beta$ for $N$ satisfying (18). Recently, in 37 it was shown that feasibility of the scenario solution to the two-step approach holds with confidence $1-\beta$ if $N$ is chosen so as to satisfy

$$
\left(\begin{array}{c}
N \\
2(d+1)
\end{array}\right)(1-\varepsilon)^{N-2(d+1)} \leq \beta
$$

which leads to the following more conservative bound for $N$ :

$$
N \geq\left\lceil\frac{2}{\varepsilon} \ln \left(\frac{1}{\beta}\right)+4(d+1)+\frac{4(d+1)}{\varepsilon} \ln \left(\frac{2}{\varepsilon}\right)\right\rceil .
$$


Remark 4. Note that the application of the scenario approach to the two-stage problem described in Remark 2 leads to a scenario based two-stage problem whose solution is not guaranteed to be feasible for the original probabilistic constraint when $N$ is chosen according to 18 , either when the constraints on the input are hard or when they are soft. As a matter of fact in both cases there is a probabilistic constraint on the output in the first stage, resulting in $\Xi=\Xi\left(\mathbf{w}^{(1)}, \mathbf{w}^{(2)}, \ldots, \mathbf{w}^{(N)}\right)$.

\section{Choice of $\mu$ and $\alpha$ and trade-off between $J$ and $h$}

The parameters $\mu$ and $\alpha$ in Problems (14)-(17) play the role of tuning parameters through which the user can modulate the trade-off between the objective of minimizing the control cost $J$ and that of having a small $h$ to strengthen the state constraint satisfaction in the system operation.

To this purpose, the two extreme cases, where all effort is put in the minimization of the sole $J$ or, viceversa, of the sole $h$, are given by the following two problems:

$$
\begin{aligned}
& \min _{\Gamma, \Theta, h} J(\Gamma, \Theta) \quad \text { subject to: } \\
& F\left(\Gamma, \Theta, h, \mathbf{w}^{(1)}, \ldots, \mathbf{w}^{(N)}\right) \leq 0
\end{aligned}
$$

and

$$
\begin{aligned}
& \min _{\Gamma, \Theta, h} h \quad \text { subject to: } \\
& F\left(\Gamma, \Theta, h, \mathbf{w}^{(1)}, \ldots, \mathbf{w}^{(N)}\right) \leq 0,
\end{aligned}
$$

where, the notation $F\left(\Gamma, \Theta, h, \mathbf{w}^{(1)}, \ldots, \mathbf{w}^{(N)}\right) \leq 0$ is used as a shorthand to refer to

$$
\left\{\begin{array} { l } 
{ | \Gamma _ { i } | + \| \Theta _ { i } \| _ { 1 } \overline { \varphi } \leq \overline { u } , i = 1 , \ldots , m M } \\
{ \| \mathbf { C x } ( \mathbf { w } ^ { ( i ) } ) \| _ { \infty } \leq h , i = 1 , \ldots , N , }
\end{array} \quad \text { or } \quad \left\{\begin{array}{l}
\left\|\mathbf{u}\left(\mathbf{w}^{(i)}\right)\right\|_{\infty} \leq \bar{u}, i=1, \ldots, N \\
\left\|\mathbf{C x}\left(\mathbf{w}^{(i)}\right)\right\|_{\infty} \leq h, i=1, \ldots, N
\end{array}\right.\right.
$$

depending on the chosen approach (hard or soft) to treat input constraints. Problem 20 corresponds to (14) or (15) with $\mu=0$. In problem 20 only the performance cost $J$ is optimized, while $h$ can be taken arbitrarily large. Problem (21) corresponds instead to $16 \mathrm{~b}$ or $17 \mathrm{~b}$ when the constraint on control cost degradation is neglected. Contrary to the previous case, in problem (21) only $h$ is minimized.

The properties below aim at showing that $\mu$ and $\alpha$ are indeed sensible tuning parameters, since by progressively increasing them over a suitable range one can explore all possible trade-off combinations between (20) and (21). Note that these properties refer to the scenario problems (14)-(17), and hold true irrespectively of the connection of (14)-(17) with the original chance-constrained problems $(9)-(12)$.

For a given $\mu$, problem

$$
\begin{aligned}
& \min _{\Gamma, \Theta, h} J(\Gamma, \Theta)+\mu h \quad \text { subject to: } \\
& F\left(\Gamma, \Theta, h, \mathbf{w}^{(1)}, \ldots, \mathbf{w}^{(N)}\right) \leq 0,
\end{aligned}
$$

which can represent either (14) or (15), may have multiple minimizers. For fixed $\mu$, we let

$$
\begin{aligned}
& \boldsymbol{J}_{\mu}=\left\{J\left(\Gamma_{\mu}, \Theta_{\mu}\right):\left(\Gamma_{\mu}, \Theta_{\mu}, h_{\mu}\right) \text { is a minimizer of } 23\right\} \\
& \left.\boldsymbol{h}_{\mu}=\left\{h_{\mu}:\left(\Gamma_{\mu}, \Theta_{\mu}, h_{\mu}\right) \text { is a minimizer of } 23\right)\right\}
\end{aligned}
$$

that is, $\boldsymbol{J}_{\mu}$ is the set of all values of the cost $J$ achieved in correspondence of the minimizers of $(23)$, while $\boldsymbol{h}_{\mu}$ is the set of all values of $h$ achieved in correspondence of the minimizers of (23). As $\mu$ is let vary, $\boldsymbol{J}_{\mu}$ and $\boldsymbol{h}_{\mu}$ are multi-valued functions of $\mu$. Similarly, for a given $\alpha$ and letting $J^{\star}$ be the optimal cost of (20), consider problem

$$
\begin{aligned}
& \min _{\Gamma, \Theta, h} h \quad \text { subject to: } \\
& \left\{\begin{array}{l}
F\left(\Gamma, \Theta, h, \mathbf{w}^{(1)}, \ldots, \mathbf{w}^{(N)}\right) \leq 0, \\
J(\Gamma, \Theta) \leq J^{\star}+\alpha
\end{array}\right.
\end{aligned}
$$


which can represent either (16) or (17). Again the problem may show multiple minimizers. We define

$$
\begin{aligned}
& \left.\boldsymbol{J}_{\alpha}=\left\{J\left(\Gamma_{\alpha}, \Theta_{\alpha}\right):\left(\Gamma_{\alpha}, \Theta_{\alpha}, h_{\alpha}\right) \text { is a minimizer of } 25\right)\right\} \\
& \left.\boldsymbol{h}_{\alpha}=\left\{h_{\alpha}:\left(\Gamma_{\alpha}, \Theta_{\alpha}, h_{\alpha}\right) \text { is a minimizer of } 25\right)\right\} .
\end{aligned}
$$

Note that in this case $\boldsymbol{h}_{\alpha}$ is a singleton for any $\alpha$. The following properties hold.

Property 1 (continuity and monotonicity I). For every $\mu \in(0,+\infty), \boldsymbol{J}_{\mu}$ and $\boldsymbol{h}_{\mu}$ are closed intervals. For $\mu_{1}<\mu_{2}$ it holds that $\max \boldsymbol{J}_{\mu_{1}} \leq \min \boldsymbol{J}_{\mu_{2}}$ and that $\min \boldsymbol{h}_{\mu_{1}} \geq \max \boldsymbol{h}_{\mu_{2}}$. Moreover,

$$
\begin{aligned}
\lim _{\mu_{1} \rightarrow \mu_{2}^{-}} \max \boldsymbol{J}_{\mu_{1}} & =\min \boldsymbol{J}_{\mu_{2},}, & \lim _{\mu_{2} \rightarrow \mu_{1}^{+}} \min \boldsymbol{J}_{\mu_{2}} & =\max \boldsymbol{J}_{\mu_{1}}, \\
\lim _{\mu_{1} \rightarrow \mu_{2}^{-}} \min \boldsymbol{h}_{\mu_{1}} & =\max \boldsymbol{h}_{\mu_{2}}, & \lim _{\mu_{2} \rightarrow \mu_{1}^{+}} \max \boldsymbol{h}_{\mu_{2}} & =\min \boldsymbol{h}_{\mu_{1}} .
\end{aligned}
$$

Note that according to Property 1 the graphs of $\boldsymbol{J}_{\mu}$ and $\boldsymbol{h}_{\mu}$ are composed by isolated vertical segments connected by single-valued increasing/decreasing curves. For a pictorial view see the illustrative plots in Fig. 1 .

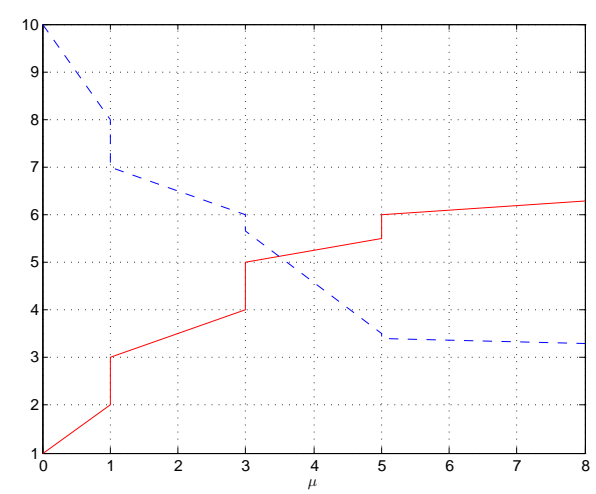

Figure 1: A possible behavior of $\boldsymbol{J}_{\mu}$ (solid line) and $\boldsymbol{h}_{\mu}$ (dashed line).

Property 2 (continuity and monotonicity II). Let $\alpha_{\infty}$ be equal to $J\left(\Gamma_{\infty}, \Theta_{\infty}\right)-J^{\star}$, where $\left(\Gamma_{\infty}, \Theta_{\infty}\right.$, $h_{\infty}$ ) denotes any minimizer of problem (21) chosen among those with the smallest value for $J$. Then, $\boldsymbol{J}_{\alpha}$ and $\boldsymbol{h}_{\alpha}$ are both single-valued continuous functions for $\alpha \in\left[0, \alpha_{\infty}\right]$. Moreover, $\boldsymbol{J}_{\alpha}$ is monotonically increasing, while $\boldsymbol{h}_{\alpha}$ is monotonically decreasing as a function of $\alpha$.

Property 3 (initial value). Let $\left(\Gamma_{0}, \Theta_{0}, h_{0}\right)$ be any minimizer of problem (20) chosen among those with the smallest value for $h$. When $\mu \rightarrow 0[\alpha \rightarrow 0], \min \boldsymbol{J}_{\mu}\left[\boldsymbol{J}_{\alpha}\right]$ tends to $J\left(\Gamma_{0}, \Theta_{0}\right)=J^{\star}$, while $\max \boldsymbol{h}_{\mu}\left[\boldsymbol{h}_{\alpha}\right]$ tends to $h_{0}$.

Property 4 (final value). Let $\alpha_{\infty}$ and $\left(\Gamma_{\infty}, \Theta_{\infty}, h_{\infty}\right)$ be as in Property Q2. When $\mu \rightarrow \infty\left[\alpha \rightarrow \alpha_{\infty}\right]$, $\max \boldsymbol{J}_{\mu}\left[\boldsymbol{J}_{\alpha}\right]$ tends to $J\left(\Gamma_{\infty}, \Theta_{\infty}\right)$, while $\min \boldsymbol{h}_{\mu}\left[\boldsymbol{h}_{\alpha}\right]$ tends to $h_{\infty}$.

Property 5 (equivalence). Let $\alpha_{\infty}$ be as in Property 2. For any $\mu \in(0,+\infty)$ and for any minimizer $\left(\Gamma_{\mu}, \Theta_{\mu}, h_{\mu}\right)$ of problem (23), there is an $\alpha \in\left(0, \alpha_{\infty}\right]$ such that $\boldsymbol{J}_{\alpha}=J\left(\Gamma_{\mu}, \Theta_{\mu}\right)$ and $\boldsymbol{h}_{\alpha}=h_{\mu}$. Conversely, for any $\alpha \in\left(0, \alpha_{\infty}\right)$ there is a $\mu \in(0,+\infty)$ and a minimizer $\left(\Gamma_{\mu}, \Theta_{\mu}, h_{\mu}\right)$ of problem 23) such that $J\left(\Gamma_{\mu}, \Theta_{\mu}\right)=$ $\boldsymbol{J}_{\alpha}$ and $h_{\mu}=\boldsymbol{h}_{\alpha}$.

The proof of Properties 145 is postponed to the next Section 6 


\subsection{Practical relevance of Properties 1 15}

In this section, we briefly discuss the importance of Properties 105 above.

Apart from Property 5, which establishes a substantial equivalence between the additional penalization term approach and the two-step approach, the other properties show that all possible trade-offs between the two extremes represented by problem $(20)$ - where cost $J$ is minimized without accounting for state restrictions - and problem (21) - where instead the state norm magnitude $h$ is minimized with no concerns about the control cost - can be achieved by increasing $\mu[\alpha]$ from 0 to $\infty$ [from 0 to $\alpha_{\infty}$ ], the dependence of both the control cost $J$ and the bound on the state norm $h$ on the tuning parameters $\mu$ and $\alpha$ being monotone and continuous (continuous in a generalized sense in the $\mu$ case). This means that $\mu$ and $\alpha$ can be indeed regarded as tuning knobs by which, as they are increased, one can give up some control performance to strengthen the restriction on the state norm.

Heuristically, one can proceed by solving the chosen program (14) or $(15)[\sqrt{16})$ or $\sqrt{17}]$ for a grid of values of $\mu$ [of $\alpha$ ], say $\mu_{1}, \mu_{2}, \ldots, \mu_{k}$ [say $\alpha_{1}, \alpha_{2}, \ldots, \alpha_{k}$ ], each time using the same realizations $\mathbf{w}^{(1)}, \mathbf{w}^{(2)}, \ldots, \mathbf{w}^{(N)}$ of the noise. This way, various solution pairs $\left(\boldsymbol{J}_{\mu_{i}}, \boldsymbol{h}_{\mu_{i}}\right)\left[\left(\boldsymbol{J}_{\alpha_{i}}, \boldsymbol{h}_{\alpha_{i}}\right)\right], i=1,2, \ldots, k$, are obtained, each showing a different trade-off between the control performance and the guarantee on the state norm. By comparing all these pairs ${ }^{2}$ the user can eventually decide which solution to buy by selecting the most suitable trade-off for the problem at hand (e.g., the user can decide whether it is better to have a smaller control cost at the price of a bigger state response or viceversa whether is preferable to have a more constrained response and a worse cost).

As is clear, one delicate point is the choice of the grid $\mu_{1}, \mu_{2}, \ldots, \mu_{k}\left[\alpha_{1}, \alpha_{2}, \ldots, \alpha_{k}\right]$, which must allow the exploration of a number of significant trade-offs. In this respect, we reckon two advantages of the two-step approach (parameter $\alpha$ ) over the additional penalization term approach (parameter $\mu$ ) as explained in the following.

1. Parameter $\alpha$ has a precise interpretation as the maximum allowed degradation of the cost, see Remark 3. As such, it is better suited to achieve predefined trade-offs between $J$ and $h$. For example, if $J^{\star}=1$, by taking a grid of values $\alpha_{1}=1 / k, \alpha_{2}=2 / k, \ldots, \alpha_{k}=k / k=1$, one knows in advance that $\mathrm{s} /$ he is going to explore all the trade-offs between $J$ and $h$ where the cost is first increased to $\boldsymbol{J}_{\alpha_{1}}=1+1 / k$ to improve the value of $\boldsymbol{h}_{\alpha_{1}}$, then to $\boldsymbol{J}_{\alpha_{2}}=1+2 / k$ to further decrease $\boldsymbol{h}_{\alpha_{2}}$, and so forth and so on.

In view of the equivalence between the two approaches, the same trade-offs between $J$ and $h$ can be obtained by means of the additional penalization term approach, but in this case no relation between the choice of the parameter $\mu$ and the variation of $J$ and $h$ is available. If, for example, $\mu_{1}, \mu_{2}, \ldots$, $\mu_{k}$ are chosen equi-spaced as the $\alpha$ 's above, it may be that the obtained values for $\boldsymbol{J}_{\mu_{1}}, \boldsymbol{J}_{\mu_{2}}, \ldots, \boldsymbol{J}_{\mu_{k}}$ and $\boldsymbol{h}_{\mu_{1}}, \boldsymbol{h}_{\mu_{2}}, \ldots, \boldsymbol{h}_{\mu_{k}}$ are all closed together, meaning that the possible trade-offs between $J$ and $h$ are not explored at all.

In conclusion, there is not an easy recipe to properly select the grid $\mu_{1}, \mu_{2}, \ldots, \mu_{k}$, while this is the case for $\alpha_{1}, \alpha_{2}, \ldots, \alpha_{k}$.

2. The additional penalization term approach has a second issue. In view of Property 2 , it may be that, in order to explore suitable trade-offs between $J$ and $h$, the user is required to grid also the vertical segments in the graphs of $\boldsymbol{J}_{\mu}$ and $\boldsymbol{h}_{\mu}$. This means that one has to distinguish among different minimizers for the same values of $\mu$, which is not trivial at all. This issue, instead, does not arise in the two-step approach, because thanks to the continuity of $\boldsymbol{J}_{\alpha}$ and $\boldsymbol{h}_{\alpha}$ (Property 1 ) all the minimizers for a given $\alpha$ returns the same trade-off between $J$ and $h$.

The sole drawback of the two-step approach is that, when probabilistic constraints on the input are also considered, the available a-priori guarantee on the feasibility of the scenario solution is weaker than that available in the other cases (compare equation $(19)$ with $(18)$ ). In this case, one may find preferable to resort to the additional penalization term approach, although, objectively, the drawback seems to be minor.

\footnotetext{
${ }^{2}$ Note that, when Theorem 1 applies, for each grid-point, the obtained approximate solution is guaranteed to be feasible for the original optimization problem except for a set of bad extractions whose probability is at most $\beta$. When considering and comparing all the approximate solutions obtained for the $k$ grid points, the guarantee holds jointly except for a set whose probability can be upper bounded by $k \cdot \beta$.
} 


\section{Proof of Properties 1,5}

For ease of exposition, in this section we prove a number of propositions, from which Properties 1.5 can be recovered as a byproduct. In particular: Property 1 follows from Propositions 1 and 7, Property 2 follows from Propositions 3 and 9 , Property 3 follows from Propositions $1,4,7$, and 9 , Property 4 follows from Propositions 5 and 9 . Property 5 follows from Propositions 6 and 10 . Note that the propositions have general validity in that they do not depend on the fact that constraint $F\left(\Gamma, \Theta, h, \mathbf{w}^{(1)}, \ldots, \mathbf{w}^{(N)}\right) \leq 0$ is as in (22). Derivations are in line with the convex analysis results in [38, though tailored to the present context.

Proposition 1. Let $\mu_{1}<\mu_{2}$ and let $\left(\Gamma_{\mu_{1}}, \Theta_{\mu_{1}}, h_{\mu_{1}}\right)$ be any solution to problem 23) when $\mu=\mu_{1}$ and let $\left(\Gamma_{\mu_{2}}, \Theta_{\mu_{2}}, h_{\mu_{2}}\right)$ be any solution when $\mu=\mu_{2}$. It holds that $J\left(\Gamma_{\mu_{1}}, \Theta_{\mu_{1}}\right) \leq J\left(\Gamma_{\mu_{2}}, \Theta_{\mu_{2}}\right)$ and that $h_{\mu_{1}} \geq h_{\mu_{2}}$. $\square$

Proof 1. Note that any solution to (23) when $\mu=\mu_{1}$ is feasible for the problem with $\mu=\mu_{2}$ and viceversa, since the constraint $F\left(\Gamma, \Theta, h, \mathbf{w}^{(1)}, \ldots, \mathbf{w}^{(N)}\right) \leq 0$ is the same for the two problems. Hence,

$$
\begin{gathered}
J\left(\Gamma_{\mu_{1}}, \Theta_{\mu_{1}}\right)+\mu_{1} h_{\mu_{1}} \leq J\left(\Gamma_{\mu_{2}}, \Theta_{\mu_{2}}\right)+\mu_{1} h_{\mu_{2}} \\
\text { [optimality of } \left.\left(\Gamma_{\mu_{1}}, \Theta_{\mu_{1}}, h_{\mu_{1}}\right)\right] \\
J\left(\Gamma_{\mu_{2}}, \Theta_{\mu_{2}}\right)+\mu_{1} h_{\mu_{2}}<J\left(\Gamma_{\mu_{2}}, \Theta_{\mu_{2}}\right)+\mu_{2} h_{\mu_{2}} \\
{\left[\mu_{1}<\mu_{2}\right]} \\
J\left(\Gamma_{\mu_{2}}, \Theta_{\mu_{2}}\right)+\mu_{2} h_{\mu_{2}} \leq J\left(\Gamma_{\mu_{1}}, \Theta_{\mu_{1}}\right)+\mu_{2} h_{\mu_{1}} \\
\text { [optimality of } \left.\left(\Gamma_{\mu_{2}}, \Theta_{\mu_{2}}, h_{\mu_{2}}\right)\right] .
\end{gathered}
$$

From 27a and 27c it follows that

$$
\mu_{1}\left(h_{\mu_{1}}-h_{\mu_{2}}\right) \leq J\left(\Gamma_{\mu_{2}}, \Theta_{\mu_{2}}\right)-J\left(\Gamma_{\mu_{1}}, \Theta_{\mu_{1}}\right) \leq \mu_{2}\left(h_{\mu_{1}}-h_{\mu_{2}}\right) .
$$

If $h_{\mu_{1}}<h_{\mu_{2}}$ from $\mu_{1}\left(h_{\mu_{1}}-h_{\mu_{2}}\right) \leq \mu_{2}\left(h_{\mu_{1}}-h_{\mu_{2}}\right)$ it would be $\mu_{2} \leq \mu_{1}$ which is in contradiction to the initial hypothesis $\mu_{1}<\mu_{2}$. Hence, $h_{\mu_{1}} \geq h_{\mu_{2}}$ and, from 28, we obtain $J\left(\Gamma_{\mu_{1}}, \Theta_{\mu_{1}}\right) \leq J\left(\Gamma_{\mu_{2}}, \Theta_{\mu_{2}}\right)$ too.

Proposition 2. Let $\left(\Gamma_{\mu}, \Theta_{\mu}, h_{\mu}\right)$ be any solution to (23). Then, $J\left(\Gamma_{\mu}, \Theta_{\mu}\right)+\mu h_{\mu}$ is a continuous strictly increasing function of $\mu$.

Proof 2. For a fixed $\mu$ even though problem (23) admits multiple solutions, the value of the optimal objective $J\left(\Gamma_{\mu}, \Theta_{\mu}\right)+\mu h_{\mu}$ is the same irrespective of the chosen solution, and, hence, $J\left(\Gamma_{\mu}, \Theta_{\mu}\right)+\mu h_{\mu}$ is indeed a single-valued function of $\mu$.

Let $\mu_{1}<\mu_{2}$ and $\left(\Gamma_{\mu_{1}}, \Theta_{\mu_{1}}, h_{\mu_{1}}\right)$ and $\left(\Gamma_{\mu_{2}}, \Theta_{\mu_{2}}, h_{\mu_{2}}\right)$ as in the proof of Proposition 1. Inequalities 27a) and 27b together show that $J\left(\Gamma_{\mu}, \Theta_{\mu}\right)+\mu h_{\mu}$ is strictly increasing. Moreover, they yield

$$
\lim _{\mu_{1} \rightarrow \mu_{2}^{-}} J\left(\Gamma_{\mu_{1}}, \Theta_{\mu_{1}}\right)+\mu_{1} h_{\mu_{1}} \leq J\left(\Gamma_{\mu_{2}}, \Theta_{\mu_{2}}\right)+\mu_{2} h_{\mu_{2}} .
$$

From 27c we have:

$$
\begin{aligned}
J\left(\Gamma_{\mu_{1}}, \Theta_{\mu_{1}}\right)+\mu_{1} h_{\mu_{1}} & \geq J\left(\Gamma_{\mu_{2}}, \Theta_{\mu_{2}}\right)+\mu_{2} h_{\mu_{2}}+\left(\mu_{1}-\mu_{2}\right) h_{\mu_{1}} \\
& \geq J\left(\Gamma_{\mu_{2}}, \Theta_{\mu_{2}}\right)+\mu_{2} h_{\mu_{2}}+\left(\mu_{1}-\mu_{2}\right) \bar{h},
\end{aligned}
$$

where $\bar{h}$ is a suitable upper bound to $h_{\mu}$ whose existence is guaranteed by the decreasing property of $h_{\mu}$ in Proposition 1, and by the fact that there exists at least one solution to problem (23) when $\mu=0$. Hence,

$$
\begin{aligned}
\lim _{\mu_{1} \rightarrow \mu_{2}^{-}} J\left(\Gamma_{\mu_{1}}, \Theta_{\mu_{1}}\right)+\mu_{1} h_{\mu_{1}} & \geq \lim _{\mu_{1} \rightarrow \mu_{2}^{-}} J\left(\Gamma_{\mu_{2}}, \Theta_{\mu_{2}}\right)+\mu_{2} h_{\mu_{2}}+\left(\mu_{1}-\mu_{2}\right) \bar{h} \\
& =J\left(\Gamma_{\mu_{2}}, \Theta_{\mu_{2}}\right)+\mu_{2} h_{\mu_{2}},
\end{aligned}
$$

which, together with 297, yields left continuity. Right continuity can be proved likewise. 
For given $\mu$ and $\alpha$, we define

$$
\begin{array}{lll}
\bar{J}_{\alpha}=\sup \boldsymbol{J}_{\alpha} & \bar{J}_{\mu}=\sup \boldsymbol{J}_{\mu} & \bar{h}_{\mu}=\sup \boldsymbol{h}_{\mu} \\
\underline{J}_{\alpha}=\inf \boldsymbol{J}_{\alpha} & \underline{J}_{\mu}=\inf \boldsymbol{J}_{\mu} & \underline{h}_{\mu}=\inf \boldsymbol{h}_{\mu},
\end{array}
$$

where $\boldsymbol{J}_{\alpha}, \boldsymbol{J}_{\mu}$, and $\boldsymbol{h}_{\mu}$ are as in $(24)$ and $(26)$. Note instead that, $\boldsymbol{h}_{\alpha}$ is a singleton for any given $\alpha, h_{\alpha}$ being the optimal objective for problem $(25)$.

Proposition 3. Both $\bar{J}_{\alpha}$ and $\underline{J}_{\alpha}$ are increasing functions of $\alpha$, while $\boldsymbol{h}_{\alpha}$ is a decreasing function of $\alpha$.

Proof 3. Let $\alpha_{1}<\alpha_{2}$. Every solution $\left(\Gamma_{\alpha_{1}}, \Theta_{\alpha_{1}}, h_{\alpha_{1}}\right)$ to problem 25 with $\alpha=\alpha_{1}$ is also feasible for the problem (25) with $\alpha=\alpha_{2}$ because $J\left(\Gamma_{\alpha_{1}}, \Theta_{\alpha_{1}}\right) \leq J^{\star}+\alpha_{1}<J^{\star}+\alpha_{2}$. Letting $\left(\Gamma_{\alpha_{2}}, \Theta_{\alpha_{2}}, h_{\alpha_{2}}\right)$ be any solution to (25) with $\alpha=\alpha_{2}$, given the optimality of $h_{\alpha_{2}}$, we have that $h_{\alpha_{2}} \leq h_{\alpha_{1}}$, i.e. $\boldsymbol{h}_{\alpha}$ is decreasing.

If $J\left(\Gamma_{\alpha_{2}}, \Theta_{\alpha_{2}}\right)>J^{\star}+\alpha_{1}$ for all the solutions to problem (25) with $\alpha=\alpha_{2}$, then $\bar{J}_{\alpha_{2}}>\bar{J}_{\alpha_{1}}$ and $\underline{J}_{\alpha_{2}}>\underline{J}_{\alpha_{1}}$ because $J\left(\Gamma_{\alpha_{1}}, \Theta_{\alpha_{1}}\right) \leq J^{\star}+\alpha_{1}$ for all the solutions to problem (25) with $\alpha=\alpha_{1}$. If, instead, $J\left(\Gamma_{\alpha_{2}}, \Theta_{\alpha_{2}}\right) \leq$ $J^{\star}+\alpha_{1}$ for some solution $\left(\Gamma_{\alpha_{2}}, \Theta_{\alpha_{2}}, h_{\alpha_{2}}\right)$ to problem [25] with $\alpha=\alpha_{2}$, then $\left(\Gamma_{\alpha_{2}}, \Theta_{\alpha_{2}}, h_{\alpha_{2}}\right)$ is feasible for problem (25) with $\alpha=\alpha_{1}$, and, since $h_{\alpha_{2}} \leq h_{\alpha_{1}}$, it is also optimal, i.e. $h_{\alpha_{2}}=h_{\alpha_{1}}$. This in turn implies that all the solutions to problem (25) with $\alpha=\alpha_{1}$ are also solutions to problem (25) with $\alpha=\alpha_{2}$. Thus, summarizing, if $J\left(\Gamma_{\alpha_{2}}, \Theta_{\alpha_{2}}\right) \leq J^{\star}+\alpha_{1}$ for some solution, then $\boldsymbol{J}_{\alpha_{1}} \subseteq \boldsymbol{J}_{\alpha_{2}}$, and, thus, $\bar{\alpha}_{\alpha_{2}} \geq \bar{J}_{\alpha_{1}}$. Moreover, since the solutions for $\alpha=\alpha_{1}$ correspond to the solutions for $\alpha=\alpha_{2}$ with the lower values for $J$, it holds that $\underline{J}_{\alpha_{2}}=\underline{J}_{\alpha_{1}}$.

Proposition 4 (initial value). Let $\left(\Gamma_{0}, \Theta_{0}, h_{0}\right)$ be a generic solution to problem 20 and let $\underline{h}_{0}=\inf h_{0}$. Any solution $\left(\Gamma_{\alpha}, \Theta_{\alpha}, h_{\alpha}\right)$ to problem (25) is such that $J\left(\Gamma_{\alpha}, \Theta_{\alpha}\right)=J\left(\Gamma_{0}, \Theta_{0}\right)=J^{\star}$ and $h_{\alpha}=h_{0}$ when $\alpha=0$.

Remark 5. Note that the solutions to problem (23) with $\mu=0$ coincide with the solutions to problem (20). In general, though $\boldsymbol{J}_{\mu}$ is a singleton when $\mu=0$, this is not so for $\boldsymbol{h}_{\mu}$ when $\mu=0$. Proposition 4 shows instead that both $\boldsymbol{J}_{\alpha}$ and $\boldsymbol{h}_{\alpha}$ are singleton when $\alpha=0$.

Proof 4. When $\alpha=0, J\left(\Gamma_{\alpha}, \Theta_{\alpha}\right)$ must be equal to $J^{\star}$ because the constraint $J(\Gamma, \Theta) \leq J^{\star}$ is forced and $J^{\star}$ is the minimal value for $J$ given the constraint $F\left(\Gamma, \Theta, h, \mathbf{w}^{(1)}, \ldots, \mathbf{w}^{(N)}\right) \leq 0$. Since any solution to 20 . is feasible for 25 with $\alpha=0$, and viceversa, then $h_{\alpha}=\underline{h}_{0}$ follows. This also shoes that $\underline{h}_{0}$ is actually a minimum.

Proposition 5 (final value). Let $\left(\Gamma_{\infty}, \Theta_{\infty}, h_{\infty}\right)$ denote a generic solution to problem $(21)$, and let $\bar{J}_{\infty}$ $=\sup \left\{J\left(\Gamma_{\infty}, \Theta_{\infty}\right)\right\}$ and $\underline{J}_{\infty}=\inf \left\{J\left(\Gamma_{\infty}, \Theta_{\infty}\right)\right\}$ where sup and inf are taken with respect to the solutions to problem (21). Then:

a) both $\underline{h}_{\mu}$ and $\bar{h}_{\mu}$ tend to $h_{\infty}$ and both $\underline{J}_{\mu}$ and $\bar{J}_{\mu}$ tend to $\underline{J}_{\infty}$ as $\mu \rightarrow \infty$.

b) for every $\alpha \geq \underline{J}_{\infty}-J^{\star}$, it holds that $\boldsymbol{h}_{\alpha}=h_{\infty}$ and that $\underline{J}_{\alpha}=\underline{J}_{\infty}$. Moreover, $\bar{J}_{\alpha}$ tends to $\bar{J}_{\infty}$ as $\alpha \rightarrow \infty$.

Remark 6. Note that $h_{\infty}$ is uniquely defined being the optimal value of (21).

Proof 5. Points a) and b) are proved in order.

a) Note that $\underline{J}_{\infty}<+\infty$ since (21) admits at least a solution. Every solution to problem (21) is also feasible for problem (23) and viceversa, since the two problems have the same constraints. Because of the minimality of $h_{\infty}$ it holds that $\bar{h}_{\mu} \geq \underline{h}_{\mu} \geq h_{\infty}$ and, hence,

$$
\lim _{\mu \rightarrow \infty} \bar{h}_{\mu} \geq \lim _{\mu \rightarrow \infty} \underline{h}_{\mu} \geq h_{\infty}
$$


(the limit exists since $\bar{h}_{\mu}$ and $\underline{h}_{\mu}$ are decreasing and bounded from below). Because of its optimality, it holds for every solution $\left(\Gamma_{\mu}, \Theta_{\mu}, h_{\mu}\right)$ to 23 that:

$$
J\left(\Gamma_{\mu}, \Theta_{\mu}\right)+\mu h_{\mu} \leq \underline{J}_{\infty}+\mu h_{\infty}
$$

which yields

$$
h_{\mu} \leq \frac{\underline{J}_{\infty}-J\left(\Gamma_{\mu}, \Theta_{\mu}\right)}{\mu}+h_{\infty} \leq \frac{\underline{J}_{\infty}}{\mu}+h_{\infty} .
$$

Hence

$$
\lim _{\mu \rightarrow \infty} \underline{h}_{\mu} \leq \lim _{\mu \rightarrow \infty} \bar{h}_{\mu} \leq \lim _{\mu \rightarrow \infty} \frac{\underline{J}_{\infty}}{\mu}+h_{\infty}=h_{\infty}
$$

which, together with 30 , yields

$$
\lim _{\mu \rightarrow \infty} \underline{h}_{\mu}=\lim _{\mu \rightarrow \infty} \bar{h}_{\mu}=h_{\infty}
$$

From (31), it also follows that:

$$
J\left(\Gamma_{\mu}, \Theta_{\mu}\right) \leq \underline{J}_{\infty}+\mu\left(h_{\infty}-h_{\mu}\right) \leq \underline{J}_{\infty}
$$

where the last inequality holds since $h_{\mu} \geq h_{\infty}$. Hence $\lim _{\mu \rightarrow \infty} \underline{J}_{\mu} \leq \lim _{\mu \rightarrow \infty} \bar{J}_{\mu} \leq \underline{J}_{\infty}$, where limits exist because $\underline{J}_{\mu}$ and $\bar{J}_{\mu}$ are increasing and bounded from above. Suppose for the sake of contradiction that

$$
\lim _{\mu \rightarrow \infty} \underline{J}_{\mu}=\tilde{J}<\underline{J}_{\infty}
$$

Letting $\tilde{\alpha}=\tilde{J}-J^{\star}$ and $\left(\Gamma_{\tilde{\alpha}}, \Theta_{\tilde{\alpha}}, h_{\tilde{\alpha}}\right)$ be a solution to problem 25 when $\alpha=\tilde{\alpha}$, it holds then that $h_{\tilde{\alpha}}=h_{\infty}$. Indeed, for every $\mu \in[0,+\infty)$ and for every solution $\left(\Gamma_{\mu}, \Theta_{\mu}, h_{\mu}\right)$ to (23), it holds that $J\left(\Gamma_{\mu}, \Theta_{\mu}\right) \leq \tilde{J}$ and hence $\left(\Gamma_{\mu}, \Theta_{\mu}, h_{\mu}\right)$ is feasible for problem (25) with $\alpha=\tilde{\alpha}$. Hence, $h_{\tilde{\alpha}} \leq h_{\mu}, \forall \mu$, which in turn implies that $h_{\tilde{\alpha}} \leq h_{\infty}=\lim _{\mu \rightarrow+\infty} h_{\mu}$. Since $h_{\infty} \leq h_{\tilde{\alpha}}$ (indeed $h_{\alpha} \geq h_{\infty} \forall \alpha$ because the set of feasible points for problem (25) is contained into the set of feasible points for (21)), we have that $h_{\tilde{\alpha}}=h_{\infty}$. The solutions to problem (25) with $\alpha=\tilde{\alpha}$ are thus feasible and optimal for problem (21). Yet, $J_{\tilde{\alpha}} \leq \tilde{J}<\underline{J}_{\infty}$, which contradicts the definition of $\underline{J}_{\infty}$. Hence necessarily

$$
\lim _{\mu \rightarrow \infty} \underline{J}_{\mu}=\underline{J}_{\infty}
$$

which also implies $\lim _{\mu \rightarrow \infty} \bar{J}_{\mu}=\underline{J}_{\infty}$.

b) The last reasoning in point a) shows that for $\alpha=\underline{J}_{\infty}-J^{\star}=\alpha_{\infty}$ any solution to problem (25) is such that $h_{\alpha}=h_{\infty}$ and hence is a solution to problem (21) as well. Moreover $J\left(\Gamma_{\alpha}, \Theta_{\alpha}\right)=\underline{J}_{\infty}$, since $J\left(\Gamma_{\alpha}, \Theta_{\alpha}\right)<\underline{J}_{\infty}$ would violate the definition of $\underline{J}_{\infty}$, while $J\left(\Gamma_{\alpha}, \Theta_{\alpha}\right)>\underline{J}_{\infty}$ would violate the condition $J\left(\Gamma_{\alpha}, \Theta_{\alpha}\right) \leq J^{\star}+\alpha$. This shows that $\underline{J}_{\infty}$ is actually a minimum.

For any fixed $\alpha \geq \underline{J}_{\infty}-J^{\star}$, all the solutions $\left(\Gamma_{\infty}, \Theta_{\infty}, h_{\infty}\right)$ to (21) such that $J\left(\Gamma_{\infty}, \Theta_{\infty}\right) \leq J^{\star}+\alpha($ at least one exists because of the definition of minimum) become feasible for problem (25) and they are optimal because $h_{\infty} \leq h_{\alpha}$, as shown in point a). Hence $h_{\alpha}=h_{\infty}$, and this in turn implies that all the solutions to 25 coincide with the solutions to 21] such that $J\left(\Gamma_{\infty}, \Theta_{\infty}\right) \leq J^{\star}+\alpha$. Thus, $\underline{J}_{\alpha}=\underline{J}_{\infty}$. Since the solutions to (21) such that $J\left(\Gamma_{\infty}, \Theta_{\infty}\right) \leq J^{\star}+\alpha$ tends to cover the whole set of solutions to (21) as $\alpha \rightarrow+\infty$, we also have that $\bar{J}_{\alpha} \rightarrow \bar{J}_{\infty}$.

This concludes the proof.

Proposition 6 (equivalence I). For every $\mu \in(0,+\infty)$ and for every solution $\left(\Gamma_{\mu}, \Theta_{\mu}, h_{\mu}\right)$ to (23) there is an $\alpha \in\left(0, \underline{J}_{\infty}-J^{\star}\right]$ such that any solution $\left(\Gamma_{\alpha}, \Theta_{\alpha}, h_{\alpha}\right)$ to [25) satisfies $J\left(\Gamma_{\alpha}, \Theta_{\alpha}\right)=J\left(\Gamma_{\mu}, \Theta_{\mu}\right)$ and $h_{\alpha}=h_{\mu}$. 
Proof 6. Take $\alpha=J\left(\Gamma_{\mu}, \Theta_{\mu}\right)-J^{\star}$ and let $\left(\Gamma_{\alpha}, \Theta_{\alpha}, h_{\alpha}\right)$ be any solution to the corresponding problem 25]. Clearly,

$$
J\left(\Gamma_{\alpha}, \Theta_{\alpha}\right) \leq J^{\star}+\alpha=J\left(\Gamma_{\mu}, \Theta_{\mu}\right) .
$$

On the other hand, the solution $\left(\Gamma_{\mu}, \Theta_{\mu}, h_{\mu}\right)$ satisfies the constraint

$$
F\left(\Gamma, \Theta, h, \mathbf{w}^{(1)}, \ldots, \mathbf{w}^{(N)}\right) \leq 0
$$

and $J\left(\Gamma_{\mu}, \Theta_{\mu}\right) \leq J^{\star}+\alpha$, so that the solution $\left(\Gamma_{\mu}, \Theta_{\mu}, h_{\mu}\right)$ is feasible for problem (25). Hence,

$$
h_{\alpha} \leq h_{\mu}
$$

because of the minimality of $h_{\alpha}$. If $J\left(\Gamma_{\alpha}, \Theta_{\alpha}\right)<J\left(\Gamma_{\mu}, \Theta_{\mu}\right)$ or $h_{\alpha}<h_{\mu}$, then we would obtain $J\left(\Gamma_{\alpha}, \Theta_{\alpha}\right)+$ $\mu h_{\alpha}<J\left(\Gamma_{\mu}, \Theta_{\mu}\right)+\mu h_{\mu}$, which is not possible because $\left(\Gamma_{\alpha}, \Theta_{\alpha}, h_{\alpha}\right)$ is feasible for 23] and $J\left(\Gamma_{\mu}, \Theta_{\mu}\right)+\mu h_{\mu}$ is optimal. Hence, $J\left(\Gamma_{\alpha}, \Theta_{\alpha}\right)=J\left(\Gamma_{\mu}, \Theta_{\mu}\right)$ and $h_{\alpha}=h_{\mu}$.

Remark 7. From the proof of Proposition $\left[6\right.$ we see that the values taken by $J\left(\Gamma_{\mu}, \Theta_{\mu}\right)$ for $\mu \in(0,+\infty)$ corresponds to the values taken by of $J\left(\Gamma_{\alpha}, \Theta_{\alpha}\right)$ when $\alpha=J\left(\Gamma_{\mu}, \Theta_{\mu}\right)-J^{\star}$. Moreover, for these $\alpha$ 's, $\boldsymbol{J}_{\alpha}$ is a singleton. By virtue of the increasing properties of $\underline{J}_{\alpha}$ and $\bar{J}_{\alpha}$ (see Proposition 3 and its proof), if for some $\alpha \in\left(0, \underline{J}_{\infty}-J^{\star}\right], \boldsymbol{J}_{\alpha}$ is a proper interval, then intermediate values are not included in any $\boldsymbol{J}_{\mu}, \mu \in(0,+\infty)$, i.e. the multi-valued function $\boldsymbol{J}_{\mu}$ is, in a sense, discontinuous.

The following proposition characterizes the conditions under which $\boldsymbol{J}_{\mu}$ shows a sort of continuity.

Proposition 7. If $J(\Gamma, \Theta)$ is convex and continuous and if the constraint $F\left(\Gamma, \Theta, h, \mathbf{w}^{(1)}, \ldots, \mathbf{w}^{(N)}\right) \leq 0$ is convex and compact, then, for every $\mu \in(0,+\infty), \boldsymbol{J}_{\mu}$ and $\boldsymbol{h}_{\mu}$ are closed intervals and for every $\mu \in[0,+\infty)$ it holds that

$$
\begin{aligned}
\lim _{\mu^{\prime} \rightarrow \mu^{+}} \bar{J}_{\mu^{\prime}}=\lim _{\mu^{\prime} \rightarrow \mu^{+}} \underline{J}_{\mu^{\prime}}=\bar{J}_{\mu} & \lim _{\mu^{\prime} \rightarrow \mu^{+}} \underline{h}_{\mu^{\prime}}=\lim _{\mu^{\prime} \rightarrow \mu^{+}} \bar{h}_{\mu^{\prime}}=\underline{h}_{\mu} \\
\lim _{\mu^{\prime} \rightarrow \mu^{-}} \underline{J}_{\mu^{\prime}}=\lim _{\mu^{\prime} \rightarrow \mu^{-}} \bar{J}_{\mu^{\prime}}=\underline{J}_{\mu} & \lim _{\mu^{\prime} \rightarrow \mu^{-}} \bar{h}_{\mu^{\prime}}=\lim _{\mu^{\prime} \rightarrow \mu^{-}} \underline{h}_{\mu^{\prime}}=\bar{h}_{\mu}
\end{aligned}
$$

Remark 8. Note that, when constraint

$$
F\left(\Gamma, \Theta, h, \mathbf{w}^{(1)}, \ldots, \mathbf{w}^{(N)}\right) \leq 0
$$

takes the expression in (22), then convexity and compactness are indeed obtained. As a matter of fact, convexity and closedness are clearly satisfied, while the constraint on the control input in 22 guarantees that $\Gamma$ and $\Theta$ take value in a bounded set, and, thanks to Propositions 2 and $4 . h \leq \underline{h}_{0}$.

Proof 7. For any given $\mu \in[0,+\infty)$, consider the sequence $\mu_{n}=\mu+\frac{1}{n}, n \in \mathbb{N}$, which tends to $\mu$ from the right and let $\left(\Gamma_{\mu_{n}}, \Theta_{\mu_{n}}, h_{\mu_{n}}\right)$ be any solution of problem (23) when $\mu=\mu_{n}$. Without any loss of generality we can assume that $\left(\Gamma_{\mu_{n}}, \Theta_{\mu_{n}}, h_{\mu_{n}}\right)$ is convergent, because if it were not, one could always extract a convergent subsequence (since the constraint set is compact) and work with the subsequence in place of the original sequence. Hence, we let

$$
\left(\Gamma^{\star}, \Theta^{\star}, h^{\star}\right)=\lim _{n \rightarrow \infty}\left(\Gamma_{\mu_{n}}, \Theta_{\mu_{n}}, h_{\mu_{n}}\right) .
$$

Thanks to the continuity of $J$, it also holds that $\lim _{n \rightarrow \infty} J\left(\Gamma_{\mu_{n}}, \Theta_{\mu_{n}}\right)=J\left(\Gamma^{\star}, \Theta^{\star}\right)$. It is a fact that $\left(\Gamma^{\star}, \Theta^{\star}, h^{\star}\right)$ is a solution of problem [23). Indeed,

$$
J\left(\Gamma^{\star}, \Theta^{\star}\right)+\mu h^{\star}=\lim _{n \rightarrow \infty}\left[J\left(\Gamma_{\mu_{n}}, \Theta_{\mu_{n}}\right)+\mu_{n} h_{\mu_{n}}\right]=J\left(\Gamma_{\mu}, \Theta_{\mu}\right)+\mu h_{\mu},
$$


where $\left(\Gamma_{\mu}, \Theta_{\mu}, h_{\mu}\right)$ is any solution to problem (23) and the last equality follows from the continuity of $J\left(\Gamma_{\mu}, \Theta_{\mu}\right)+\mu h_{\mu}$ as a function of $\mu$ (see Proposition 2). Furthermore, we have that

$$
J\left(\Gamma^{\star}, \Theta^{\star}\right)=\bar{J}_{\mu}, \quad h^{\star}=\underline{h}_{\mu},
$$

i.e. $\left(\Gamma^{\star}, \Theta^{\star}, h^{\star}\right)$ is the solution of problem (23) where the extremal values $\bar{J}_{\mu}$ and $\underline{h}_{\mu}$ are achieved. As a matter of fact, if this was not the case, then there would be a solution $\left(\Gamma_{\mu}, \Theta_{\mu}, h_{\mu}\right)$ such that $J\left(\Gamma^{\star}, \Theta^{\star}\right)<$ $J\left(\Gamma_{\mu}, \Theta_{\mu}\right)$ and/or $h^{\star}>h_{\mu}$, in contradiction with the fact that $J\left(\Gamma_{\mu_{n}}, \Theta_{\mu_{n}}\right) \rightarrow J\left(\Gamma^{\star}, \Theta^{\star}\right)$ and $J\left(\Gamma_{\mu_{n}}, \Theta_{\mu_{n}}\right) \geq$ $J\left(\Gamma_{\mu}, \Theta_{\mu}\right)$ and with the fact that $h_{\mu_{n}} \rightarrow h^{\star}$ and $h_{\mu_{n}} \leq h_{\mu}$ (see Proposition 1). Hence, $\lim _{n \rightarrow \infty} J\left(\Gamma_{\mu_{n}}, \Theta_{\mu_{n}}\right)=$ $\bar{J}_{\mu}$ and $\lim _{n \rightarrow \infty} h_{\mu_{n}}=\underline{h}_{\mu}$, and, since $\bar{J}_{\mu} \leq \underline{J}_{\mu_{n}} \leq \bar{J}_{\mu_{n}} \leq J\left(\Gamma_{\mu_{n-1}}, \Theta_{\mu_{n-1}}\right)$ and $\underline{h}_{\mu} \geq \bar{h}_{\mu_{n}} \geq \underline{h}_{\mu_{n}} \geq h_{\mu_{n-1}}$, (32) remains proven.

Similarly, for $\mu>0$, one can consider the sequence $\mu_{m}=\mu-\frac{1}{m}, m \in \mathbb{N}$, and the corresponding sequence of solutions $\left(\Gamma_{\mu_{m}}, \Theta_{\mu_{m}}, h_{\mu_{m}}\right)$, which without loss of generality can be assumed to be convergent. Letting

$$
\left(\Gamma_{\star}, \Theta_{\star}, h_{\star}\right)=\lim _{m \rightarrow \infty}\left(\Gamma_{\mu_{m}}, \Theta_{\mu_{m}}, h_{\mu_{m}}\right),
$$

it can be proved likewise as before that $J\left(\Gamma_{\mu_{m}}, \Theta_{\mu_{m}}\right) \rightarrow J\left(\Gamma_{\star}, \Theta_{\star}\right)$, that $\left(\Gamma_{\star}, \Theta_{\star}, h_{\star}\right)$ is a solution to problem 23), and that $J\left(\Gamma_{\star}, \Theta_{\star}\right)=\underline{J}_{\mu}$ and $h_{\star}=\bar{h}_{\mu}$. Hence, $\lim _{m \rightarrow \infty} J\left(\Gamma_{\mu_{m}}, \Theta_{\mu_{m}}\right)=\underline{J}_{\mu}$ and $\lim _{m \rightarrow \infty} h_{\mu_{m}}=\bar{h}_{\mu}$, and again (33) remains proven because $J\left(\Gamma_{\mu_{m-1}}, \Theta_{\mu_{m-1}}\right) \leq \underline{J}_{\mu_{m}} \leq \bar{J}_{\mu_{m}} \leq \bar{J}_{\mu}$ and $h_{\mu_{m-1}} \geq \bar{h}_{\mu_{m}} \geq \underline{h}_{\mu_{m}} \geq$ $\underline{h}_{\mu}$.

To prove the remaining part of the proposition, let $\left(\Gamma_{\lambda}, \Theta_{\lambda}, h_{\lambda}\right)=\lambda\left(\Gamma^{\star}, \Theta^{\star}, h^{\star}\right)+(1-\lambda)\left(\Gamma_{\star}, \Theta_{\star}, h_{\star}\right)$, $\lambda \in[0,1]$. Given the convexity of the constraint set, $\left(\Gamma_{\lambda}, \Theta_{\lambda}, h_{\lambda}\right)$ is feasible for problem (23) and, moreover, it is also a solution. As a matter of fact,

$$
\begin{aligned}
J & \left(\Gamma_{\lambda}, \Theta_{\lambda}\right)+\mu h_{\lambda} \\
& =J\left(\lambda \Gamma^{\star}+(1-\lambda) \Gamma_{\star}, \lambda \Theta^{\star}+(1-\lambda) \Theta_{\star}\right)+\mu\left[\lambda h^{\star}+(1-\lambda) h_{\star}\right] \\
& \leq[J \text { is convex }] \\
& \leq \lambda J\left(\Gamma^{\star}, \Theta^{\star}\right)+(1-\lambda) J\left(\Gamma_{\star}, \Theta_{\star}\right)+\lambda \mu h^{\star}+(1-\lambda) \mu h_{\star} \\
& =\lambda\left[J\left(\Gamma^{\star}, \Theta^{\star}\right)+\mu h^{\star}\right]+(1-\lambda)\left[J\left(\Gamma_{\star}, \Theta_{\star}\right)+\mu h_{\star}\right] \\
& =\left[\operatorname{since} J\left(\Gamma^{\star}, \Theta^{\star}\right)+\mu h^{\star}=J\left(\Gamma_{\star}, \Theta_{\star}\right)+\mu h_{\star}=J\left(\Gamma_{\mu}, \Theta_{\mu}\right)+\mu h_{\mu}\right] \\
& =J\left(\Gamma_{\mu}, \Theta_{\mu}\right)+\mu h_{\mu},
\end{aligned}
$$

and being $J\left(\Gamma_{\mu}, \Theta_{\mu}\right)+\mu h_{\mu}$ optimal, it must be that $J\left(\Gamma_{\lambda}, \Theta_{\lambda}\right)+\mu h_{\lambda}=J\left(\Gamma_{\mu}, \Theta_{\mu}\right)+\mu h_{\mu}$. This also implies that, since $h_{\lambda}=\lambda h^{\star}+(1-\lambda) h_{\star}$ by definition, $J\left(\Gamma_{\lambda}, \Theta_{\lambda}\right)=\lambda J\left(\Gamma^{\star}, \Theta^{\star}\right)+(1-\lambda) J\left(\Gamma_{\star}, \Theta_{\star}\right)$, which is to say that $J\left(\Gamma_{\lambda}, \Theta_{\lambda}\right)$ takes on all the values between $\underline{J}_{\mu}$ and $\bar{J}_{\mu}$ and $h_{\lambda}$ takes on all the values between $\underline{h}_{\mu}$ and $\bar{h}_{\mu}$ as $\lambda$ is let vary in $[0,1]$. That is, $\boldsymbol{J}_{\mu}$ and $\boldsymbol{h}_{\mu}$ are closed intervals.

Note that if $J(\Gamma, \Theta)$ is continuous and strictly convex, then Proposition 7 holds true without assuming that $F\left(\Gamma, \Theta, h, \mathbf{w}^{(1)}, \ldots, \mathbf{w}^{(N)}\right) \leq 0$ is compact. Indeed, thanks to Proposition 5 , the optimal $\Gamma_{\mu}, \Theta_{\mu}$ has to lie in the level set $\left\{(\Gamma, \Theta): J(\Gamma, \Theta) \leq \underline{J}_{\infty}\right\}$, which is a compact set thanks to the strict convexity of $J$, while $h_{\mu} \leq \underline{h}_{0}$ as already noticed. When $J$ is as in (7), strict convexity can be secured, e.g., by assuming that matrices $\mathbf{R}$ and $V_{\varphi \varphi}=\mathbb{E}\left[(\varphi(\mathbf{w})-\mathbb{E}[\varphi(\mathbf{w})])\left(\varphi(\mathbf{w})-\mathbb{E}[\varphi(\mathbf{w}))^{T}\right]\right.$ are positive definite. See Appendix Appendix A for a proof.

Under the assumption that $J$ is strictly convex the following stronger proposition holds.

Proposition 8. If $J(\Gamma, \Theta)$ is continuous and strictly convex and

$$
F\left(\Gamma, \Theta, h, \mathbf{w}^{(1)}, \ldots, \mathbf{w}^{(N)}\right) \leq 0
$$

is a convex constraint then $\boldsymbol{J}_{\mu}$ and $\boldsymbol{h}_{\mu}$ are continuous single-valued functions over $(0,+\infty)$. 
Proof 8. As remarked above, under the present assumptions, the same argument used to prove Proposition 7 applies. However, because of strict convexity, if $\left(\Gamma^{\star}, \Theta^{\star}, h^{\star}\right) \neq\left(\Gamma_{\star}, \Theta_{\star}, h_{\star}\right)$, then (34) would write

$$
J\left(\Gamma_{\lambda}, \Theta_{\lambda}\right)+\mu h_{\lambda}<J\left(\Gamma_{\mu}, \Theta_{\mu}\right)+\mu h_{\mu},
$$

which contradicts the minimality of $J\left(\Gamma_{\mu}, \Theta_{\mu}\right)+\mu h_{\mu}$. Hence necessarily

$$
\left(\Gamma^{\star}, \Theta^{\star}, h^{\star}\right)=\left(\Gamma_{\star}, \Theta_{\star}, h_{\star}\right),
$$

showing that $\boldsymbol{J}_{\mu}$ and $\boldsymbol{h}_{\mu}$ are continuous and single-valued.

Proposition 9. Let $\underline{J}_{\infty}$ as in Proposition 5. Under the assumptions of Proposition 7 and for $\alpha \in\left[0, \underline{J}_{\infty}-\right.$ $\left.J^{\star}\right], \boldsymbol{J}_{\alpha}$ and $\boldsymbol{h}_{\alpha}$ are continuous single-valued functions.

Proof $\mathbf{9} . \boldsymbol{h}_{\alpha}$ is single-valued by definition. $\boldsymbol{J}_{\alpha}$ has been already shown to be single-valued for $\alpha=0$ and for $\alpha=\underline{J}_{\infty}-J^{\star}$ in Propositions 4 and 5, respectively, while for $\alpha \in\left(0, \underline{J}_{\infty}-J^{\star}\right)$ the property follows in view of Proposition 6 (Remark 7) and Proposition [7. Proposition 6 also yields that for $\alpha \in\left[0, \underline{J}_{\infty}-J^{\star}\right]$ the image of $\boldsymbol{J}_{\alpha}$ is $\left[J^{\star}, \underline{J}_{\infty}\right]$ while the image of $\boldsymbol{h}_{\alpha}$ is $\left[h_{\infty}, \underline{h}_{0}\right]$, where $\underline{h}_{0}$ is as in 四. Continuity follows from the monotonicity of $\boldsymbol{J}_{\alpha}$ and $\boldsymbol{h}_{\alpha}$ stated in Proposition 3.

Eventually, we have also the following proposition, which, together with Proposition 6, shows a substantial equivalence between problems (25) and 23].

Proposition 10 (equivalence II). Under the assumption of Proposition 7 , for every $\alpha \in\left(0, J_{\infty}-J^{\star}\right)$ there is a $\mu \in(0,+\infty)$ and a solution $\left(\Gamma_{\mu}, \Theta_{\mu}, h_{\mu}\right)$ to problem (23) such that $J\left(\Gamma_{\mu}, \Theta_{\mu}\right)=\boldsymbol{J}_{\alpha}$ and $h_{\mu}=\boldsymbol{h}_{\alpha}$.

Proof 10. For any given $\alpha \in\left(0, \underline{J}_{\infty}-J^{\star}\right), \boldsymbol{J}_{\alpha} \in\left(J^{\star}, \underline{J}_{\infty}\right), \boldsymbol{h}_{\alpha} \in\left(\underline{h}_{0}, h_{\infty}\right)$. By Proposition 7 , we have that

$$
\exists \mu, \exists\left(\Gamma_{\mu}, \Theta_{\mu}, h_{\mu}\right): J\left(\Gamma_{\mu}, \Theta_{\mu}\right)=\boldsymbol{J}_{\alpha} .
$$

The solution $\left(\Gamma_{\mu}, \Theta_{\mu}, h_{\mu}\right)$ to problem (23) is feasible for problem (25) and, hence, $\boldsymbol{h}_{\alpha} \leq h_{\mu}$. On the other hand, $\boldsymbol{h}_{\alpha}<h_{\mu}$ would give for some solution $\left(\Gamma_{\alpha}, \Theta_{\alpha}, h_{\alpha}\right)$

$$
J\left(\Gamma_{\alpha}, \Theta_{\alpha}\right)+\mu h_{\alpha}=\boldsymbol{J}_{\alpha}+\mu \boldsymbol{h}_{\alpha}=J\left(\Gamma_{\mu}, \Theta_{\mu}\right)+\mu \boldsymbol{h}_{\alpha}<J\left(\Gamma_{\mu}, \Theta_{\mu}\right)+\mu h_{\mu},
$$

which is an absurd because any solution to problem (25) is feasible for problem (23) and the right-hand side of (35) is the optimal value for problem 23). Hence, $h_{\mu}=\boldsymbol{h}_{\alpha}$.

\section{Numerical example} 24.

The approaches described in Sections 3.1 and 3.2 are here applied to a numerical example inspired by

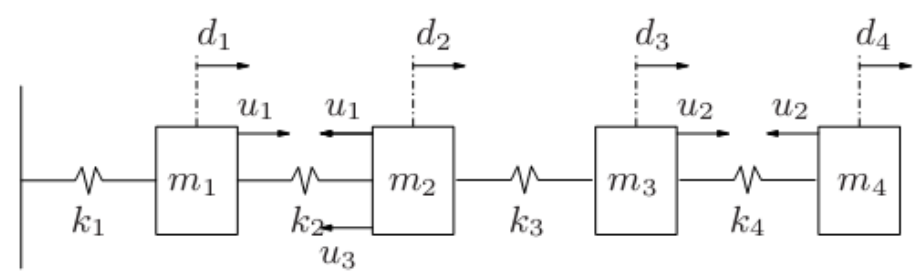

Figure 2: Scheme of the mechanical system. 
We consider the mechanical system reported in Figure 2, which is composed of four masses and four springs. The state of the system is given by the mass displacements from the equilibrium position when all inputs are zero and their derivatives: $x=\left[d_{1}, d_{2}, d_{3}, d_{4}, \dot{d}_{1}, \dot{d}_{2}, \dot{d}_{3}, \dot{d}_{4}\right]^{T}$. The control input is $u=\left[u_{1}, u_{2}, u_{3}\right]^{T}$ where $u_{1}, u_{2}$ and $u_{3}$ are forces acting on the masses as in Figure 2.

We set all masses and stiffness constants equal to 1 , i.e., $m_{1}=m_{2}=m_{3}=m_{4}=1$ and $k_{1}=k_{2}=k_{3}=$ $k_{4}=1$, and consider the discrete time model of the system

$$
x_{t+1}=A x_{t}+B u_{t}+w_{t},
$$

obtained by time discretization under the assumption that the control action is piecewise constant over the intervals $[t, t+1)$ and where the state of the system is supposed to be affected by a white Gaussian noise $w$ with zero mean and covariance matrix $I_{8 \times 8}$.

Our goal is to design a state feedback control policy that is able to counteract the disturbance $w$, maintaining the third and the fourth masses close to their equilibrium positions and keeping the springs within their linear operating domain. The latter requirement is explicitly accounted for by imposing a constraint on the spring deformations.

To the purpose of regulating the third and the fourth masses around their equilibrium positions, we consider the average control cost (4) with a prediction horizon of length $M=5$ and constant weight matrices

$$
Q=\left[\begin{array}{cc|c}
I_{2 \times 2} 10^{-3} & 0_{2 \times 2} & 0_{4 \times 4} \\
0_{2 \times 2} & I_{2 \times 2} & \\
\hline 0_{4 \times 4} & & 0_{4 \times 4}
\end{array}\right] \quad R=10^{-6} I_{3 \times 3}
$$

Let

so that

$$
C=\left[\begin{array}{cccc|c}
1 & 0 & 0 & 0 & \\
-1 & 1 & 0 & 0 & 0_{4 \times 4} \\
0 & -1 & 1 & 0 & \\
0 & 0 & -1 & 1 &
\end{array}\right]
$$

sothat

$$
C x_{i}=\left[\begin{array}{c}
d_{1, i} \\
d_{2, i}-d_{1, i} \\
d_{3, i}-d_{2, i} \\
d_{4, i}-d_{3, i}
\end{array}\right]
$$

provides the springs deformation at time $i$. Then, the state constraints, introduced to limit the springs deformation, can be expressed as

$$
\|\mathbf{C x}\|_{\infty} \leq h
$$

where $\mathbf{C}$ is defined as in 8 . Eventually, we suppose that the control input is subject to the saturation limit

$$
\|\mathbf{u}\|_{\infty} \leq \bar{u}
$$

where $\bar{u}=4$.

We shall now illustrate the performance of the approaches in Sections 3.1 and 3.2 . The control policy is parameterized according to (2) where $\varphi(\cdot)$ is the saturation function in (3) with $\bar{\varphi}$ set equal to 2 . The initial state is zero, i.e., the system starts at the equilibrium point. We shall focus on the case when constraints on both the control input and the state are expressed in probability with an admissible violation $\varepsilon=0.1$. In the scenario solution to the resulting chance-constrained optimization problems, we set $\beta=10^{-5}$. Correspondingly, the number of disturbance realizations to extract is $N=3455$. All scenario problems were solved by running YALMIP over SeDuMi 31.

Table 1 reports the optimal values of $J$ and $h$ obtained by the approach in Section 3.1 where the penalized control cost $J^{\prime}=J+\mu h$ is adopted for five different values of $\mu$. The last column of Table 1 also reports the estimate $\hat{\varepsilon}$ of the actual probability of constraint violation calculated through the Monte Carlo method over 
5000 runs of the controlled system. It is worth noticing that the estimate $\hat{\varepsilon}$ is always smaller than $\varepsilon=0.1$, as it is guaranteed by the scenario theory with confidence $1-5 \cdot 10^{-5}$.

From Table 1 it is apparent that parameter $\mu$ affects the trade-off between the two objectives, i.e., control cost and state constraints: for small values of $\mu$, the state constraints are ineffective in practice, whereas for large $\mu$ 's, $h$ decreases at the price of a significant increase of $J$. Results reported in Table 2 refer

Table 1: Results of the approach with additional penalization term in the control cost.

\begin{tabular}{|r|r|r|r|}
\hline$\mu$ & $J$ & $h$ & $\hat{\varepsilon}$ \\
\hline 0 & 20.41 & 12.60 & 0.0366 \\
0.1 & 20.50 & 10.40 & 0.0394 \\
1 & 21.69 & 7.58 & 0.0452 \\
10 & 28.02 & 5.84 & 0.0546 \\
100 & 36.78 & 5.40 & 0.0724 \\
\hline
\end{tabular}

to the two-step approach in Section 3.2. The same comments as for Table 1 apply. In particular, the actual constraint violation $\hat{\varepsilon}$ is small and lower than $\varepsilon=0.1$. This is a quite interesting fact, since there are no a-priori guarantees on the feasibility of the scenario solution in this case for $N$ satisfying (18) as discussed in Section 4.2

Table 2: Results of the two-step approach.

\begin{tabular}{|r|r|r|r|}
\hline$\alpha / J^{\star}$ & $J$ & $h$ & $\hat{\varepsilon}$ \\
\hline 0 & 20.41 & 12.60 & 0.0366 \\
0.05 & 21.43 & 7.86 & 0.0460 \\
0.1 & 22.45 & 6.99 & 0.0500 \\
0.2 & 24.49 & 6.35 & 0.0536 \\
0.5 & 30.62 & 5.64 & 0.0654 \\
\hline
\end{tabular}

Note that, since we are imposing a probabilistic bound on the control input $\mathbf{u}$, there may be disturbance realizations such that the bound on $\mathbf{u}$ is violated. If this is the case in the simulations of the controlled system, the components of $\mathbf{u}$ whose absolute value exceeds $\bar{u}$ are saturated to $\pm \bar{u}$ (clipping of the control input). It is then interesting to assess the performance of the clipped version of the obtained controllers. To this purpose, the average control cost (4) when clipping holds, say $\hat{J}$, is estimated via Monte Carlo simulations over 5000 runs and is reported in Table 3 . As it appears, the values of $\hat{J}$ are quite close to the

Table 3: Average control cost for the clipped controllers

\begin{tabular}{|r|r|r||r|r|r|}
\hline$\mu$ & $J$ & $\hat{J}$ & $\alpha / J^{\star}$ & $J$ & $\hat{J}$ \\
\hline 0 & 20.41 & 20.66 & 0 & 20.41 & 20.66 \\
0.1 & 20.50 & 20.75 & 0.05 & 21.43 & 21.71 \\
1 & 21.69 & 21.98 & 0.1 & 22.45 & 22.74 \\
10 & 28.02 & 28.40 & 0.2 & 24.49 & 24.84 \\
100 & 36.78 & 37.18 & 0.5 & 30.62 & 31.84 \\
\hline
\end{tabular}

corresponding values of $J$ in Tables 1 and 2 . This is not surprising given that the violation of the constraint on the input has small probability to occur, which makes the impact of clipping on performance negligible. This is not the case for the optimal LQG control policy where constraints on both state and input are ignored. Clipped LQG control has a cost $\hat{J}=36.09$, with a significant degradation with respect to the optimal LQG cost $J=13.81$. 
As for the constraint on the state, Figures 3 and 4 depict the probability distribution function of $\|\mathbf{C x}\|_{\infty}$ for the designed clipped controllers in correspondence of the different values of $\mu$ and $\alpha$, respectively, together with the probability distribution of $\|\mathbf{C x}\|_{\infty}$ for the clipped LQG control.

These figures reveal that all designed clipped controllers outperform the clipped LQG policy in terms of state constraint guarantees. Moreover, Figures 3 and 4 together with Table 3 show that as $\mu$ and $\alpha$ vary, a trade-off between performance and state constraint guarantees similar to that revealed by Tables 1 and 2 is achieved when clipping is active.

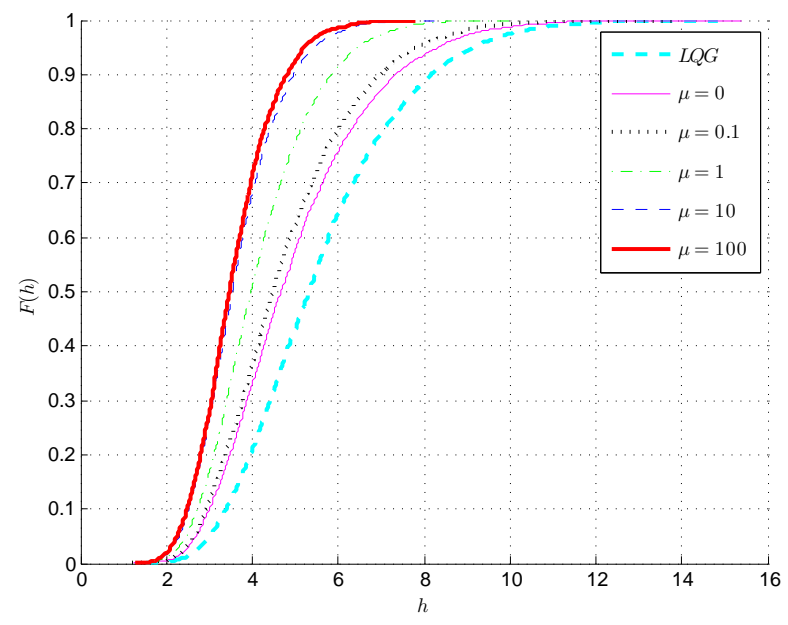

Figure 3: Probability distribution function of $\|\mathbf{C x}\|_{\infty}$ for the clipped controllers corresponding to the different values of $\mu$ and for the clipped LQG controller.

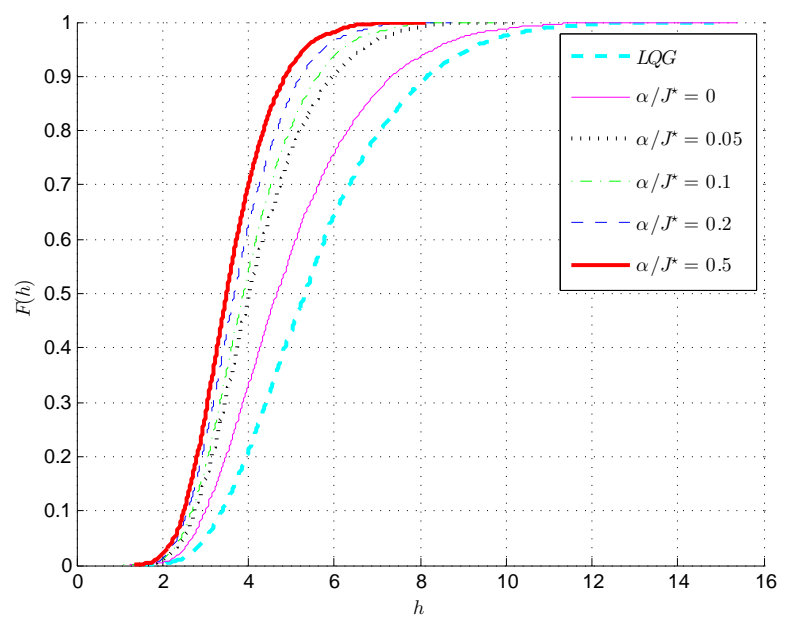

Figure 4: Probability distribution function of $\|\mathbf{C x}\|_{\infty}$ for the clipped controllers corresponding to the different values of $\alpha$ and for the clipped LQG controller. 


\section{Conclusion}

In this paper, we proposed a control design methodology to address finite-horizon control for a stochastic linear system subject to a possible unbounded disturbance. The presence of constraints, and in particular of the constraints in probability on the state variables, makes the problem difficult to solve. In order to guarantee state constraint feasibility while optimizing the control performance, we define appropriate parametric chance-constrained optimization problems where different trade-off levels between the minimization of the control cost and the satisfaction of the state constraint can be explored by tuning some parameter. A numerical example shows the efficacy of the approach. Notably, sampling of the constraints in probability allows to obtain a computationally affordable solution while retaining chance-constraints feasibility.

The proposed approach to stochastic constrained control is amenable for a receding-horizon implementation. However, long-run properties of the obtained solution are not considered here and deserve further study.

\section{References}

[1] E. Camacho, C. Bordons, Model predictive Control Theory and Design, Springer Verlag, 2004.

[2] J. Rawlings, D. Mayne, Model predictive Control Theory and Design, Nob Hill publishing, 2009.

[3] P. Goulart, E. Kerrigan, J. Maciejowski, Optimization over state feedback policies for robust control with constraints, Automatica 42 (4) (2006) 523-533.

[4] P. Hokayem, D. Chatterjee, J. Lygeros, On stochastic model predictive control with bounded control inputs, in: IEEE Conference on Decision and Control, 2009.

[5] P. Hokayem, E. Cinquemani, D. Chatterjee, F. Ramponi, J. Lygeros, Stochastic receding horizon control with output feedback and bounded controls, Automatica 48 (1) (2012) 77-88.

[6] G. Calafiore, M. Campi, Uncertain convex programs: randomized solutions and confidence levels, Mathematical Programming 102 (1) (2005) 25-46.

[7] G. Calafiore, M. Campi, The scenario approach to robust control design, IEEE Transactions on Automatic Control 51 (5) (2006) $742-753$

[8] M. Campi, S. Garatti, The exact feasibility of randomized solutions of uncertain convex programs, SIAM Journal on Optimization 19 (3) (2008) 1211-1230.

[9] M. Campi, S. Garatti, M. Prandini, The scenario approach for systems and control design, Annual Reviews in Control 33 (2) (2009) 149-157.

[10] R. Tempo, G. Calafiore, F. Dabbene, Randomized Algorithms for Analysis and Control of Uncertain Systems, with Applications, Springer-Verlag, London, UK, 2013.

[11] L. Deori, S. Garatti, M. Prandini, Stochastic constrained control: Trading performance for state constraint feasibility, in: Proceedings of the 2013 European Control Conference, 2013, pp. 2740-2745.

[12] G. Calafiore, L. Fagiano, Robust model predictive control via random convex programming, in: Decision and Control and European Control Conference (CDC-ECC), 2011 50th IEEE Conference on, 2011, pp. 1910-1915.

[13] G. Schildbach, G. Calafiore, L. Fagiano, M. Morari, Randomized Model Predictive Control for Stochastic Linear Systems, in: American Control Conference, Montreal, Canada, 2012, pp. 417-422.

[14] G. Calafiore, L. Fagiano, Robust model predictive control via scenario optimization, IEEE Transactions on Automatic Control 58 (1) (2013) 219-224.

[15] S. Qin, T. Badgwell, A survey of industrial model predictive control technology, Control Engineering Practice 11 (7) (2003) 733-764. doi:10.1016/s0967-0661(02)00186-7.

[16] I. Batina, A. Stoorvogel, S. Weiland, Optimal control of linear, stochastic systems with state and input constraints, in: Proc. of the 41st IEEE Conference on Decision and Control, 2002.

[17] D. V. Hessem, O. Bosgra, Stochastic closed-loop model predictive control of continuous nonlinear chemical processes, Journal of Process Control 16 (3) (2006) $225-241$.

[18] D. Bertsimas, D. Brown, Constrained stochastic LQC: A tractable approach, IEEE Transactions on Automatic Control $52(10)(2007) 1826-1841$

[19] J. Primbs, A soft constraint approach to stochastic receding horizon control, in: Proc. of the 46th IEEE Conference on Decision and Control, 2007.

[20] M. Ono, B. Williams, Iterative risk allocation: A new approach to robust model predictive control with a joint chance constraint, in: Proc. of the 47th IEEE Conference on Decision and Control, 2008.

[21] J. Primbs, H. Chang, Stochastic receding horizon control of constrained linear systems with state and control multiplicative noise, IEEE Transactions on Automatic Control 54 (2) (2009) 221-230.

22] L. Blackmore, M. Ono, Convex chance constrained predictive control without sampling, Proceedings of the AIAA Guidance, Navigation and Control Conference.

[23] M. Cannon, B. Kouvaritakis, X. Wu, Probabilistic constrained mpc for multiplicative and additive stochastic uncertainty, IEEE Transactions on Automatic Control 54 (2009) 1626-1632.

[24] E. Cinquemani, M. Agarwal, D. Chatterjee, J. Lygeros, Convexity and convex approximations of discrete-time stochastic control problems with constraints, Automatica 47 (9) (2011) 2082-2087. 
[25] M. Farina, L. Giulioni, L. Magni, R. Scattolini, An approach to output-feedback mpc of stochastic linear discrete-time systems, Automatica 55 (2015) 140-149.

[26] D. Q. Mayne, Model predictive control: Recent developments and future promise, Automatica 50 (12) (2014) 2967 - 2986. doi:http://dx.doi.org/10.1016/j.automatica.2014.10.128

URL http://www.sciencedirect.com/science/article/pii/S0005109814005160

[27] A. Mesbah, Stochastic model predictive control: An overview and perspectives for future research, IEEE Control Systems MagazineTo appear.

[28] D. Chatterjee, F. Ramponi, P. Hokayem, J. Lygeros, On mean square boundedness of stochastic linear systems with bounded controls Systems \& Control Letters 61 (2) (2012) 375 - 380. doi:http://dx.doi.org/10.1016/j.sysconle. 2011.12 .002

URL http://www.sciencedirect.com/science/article/pii/S016769111100301X

[29] M. Lorenzen, F. Dabbene, R. Tempo, F. Allgower, Constraint-Tightening and Stability in Stochastic Model Predictive Control, arXiv:1511.03488.

[30] M. Grant, S. Boyd, CVX: Matlab software for disciplined convex programming, version 1.21, http://cvxr.com/cvx (Feb 2011).

[31] J. Löfberg, Yalmip: a toolbox for modeling and optimization in MATLAB in: Proc. of the CACSD Conference, Taipei, Taiwan, 2004. URL http://control.ee.ethz.ch/ joloef/yalmip.php

[32] T. Alamo, R. Tempo, A. Luque, On the sample complexity of randomized approaches to the analysis and design under uncertainty, in: Proc. of the 2010 Amer. Contr. Conf., Baltimore, MD, USA, 2010, pp. 4671-4676.

[33] T. Alamo, R. Tempo, A. Luque, D. R. Ramirez, Randomized methods for design of uncertain systems: Sample complexity and sequential algorithms, Automatica 52 (2015) 160-172.

[34] M. Campi, S. Garatti, A sampling-and-discarding approach to chance-constrained optimization: Feasibility and optimality, Journal of Optimization Theory and Applications 148 (2) (2011) 257-280.

[35] T. Alamo, R. Tempo, E. F. Camacho, A randomized strategy for probabilistic solutions of uncertain feasibility and optimization problems, IEEE Transactions on Automatic Control 54 (2009) 2545-2559.

[36] S. Grammatico, X. Zhang, K. Margellos, P. Goulart, J. Lygeros, A scenario approach for non-convex control design, IEEE Transactions on Automatic Control 61 (2) (2016) 334-345.

[37] K. Margellos, M. Prandini, J. Lygeros, On the connection between compression learning and scenario based single-stage and cascading optimization problems, IEEE Transactions on Automatic Control 60 (10) (2015) $2716-2721$.

[38] R. Rockafellar, Convex Analysis, Princeton University Press, 1997.

\section{Appendix A. Strict convexity of the cost function}

Proposition 11. If matrices $\mathbf{R}$ and

$$
V_{\varphi \varphi}=\mathbb{E}\left[(\varphi(\mathbf{w})-\mathbb{E}[\varphi(\mathbf{w})])(\varphi(\mathbf{w})-\mathbb{E}[\varphi(\mathbf{w})])^{T}\right]
$$

are positive definite, then the cost function (4) is strictly convex.

Proof 11. Being $J(\Gamma, \Theta)$ the sum of two quadratic functions (see (7)) it is enough to show that one of them is strictly convex. We consider

$$
\mathbb{E}\left[\mathbf{u}^{T} \mathbf{R u}\right]=\left(\Gamma+\Theta m_{\varphi}\right)^{T} \mathbf{R}\left(\Gamma+\Theta m_{\varphi}\right)+\operatorname{tr}\left(\mathbf{R}^{\frac{1}{2}} \Theta V_{\varphi \varphi} \Theta^{T} \mathbf{R}^{\frac{1}{2}}\right) .
$$

Strict convexity is equivalent to the property:

$$
\mathbb{E}\left[\mathbf{u}^{T} \mathbf{R u}\right]=0 \Longleftrightarrow \Gamma=0 \Theta=0
$$

Since $\mathbb{E}\left[\mathbf{u}^{T} \mathbf{R u}\right]$ is the sum of two non negative terms, it is null if and only if both terms are null. Since $\mathbf{R}$ is positive definite the first term is null if and only if

$$
\Gamma+\Theta m_{\varphi}=0
$$

The second term in A.1 can be rewritten as

$$
\operatorname{tr}\left(\mathbf{R}^{\frac{1}{2}} \Theta V_{\varphi \varphi} \Theta^{T} \mathbf{R}^{\frac{1}{2}}\right)=\sum_{i=1}^{M m}\left(\mathbf{R}^{\frac{1}{2}} \Theta\right)_{i} V_{\varphi \varphi}\left(\mathbf{R}^{\frac{1}{2}} \Theta\right)_{i}^{T}
$$


where $\left(\mathbf{R}^{\frac{1}{2}} \Theta\right)_{i}$ denotes the $i$-th row of $\mathbf{R}^{\frac{1}{2}} \Theta$. Since $V_{\varphi \varphi}$ is positive definite then

$$
\operatorname{tr}\left(\mathbf{R}^{\frac{1}{2}} \Theta V_{\varphi \varphi} \Theta^{T} \mathbf{R}^{\frac{1}{2}}\right)=0 \Longleftrightarrow\left(\mathbf{R}^{\frac{1}{2}} \Theta\right)_{i}=0 \forall i=1, \ldots, M m \Longleftrightarrow \mathbf{R}^{\frac{1}{2}} \Theta=0 .
$$

Since $\mathbf{R}$ is positive definite this implies that $\Theta=0$ and from $\mathrm{A} .2$ it follows that $\Gamma=0$ as well. 